\title{
THE MAINTENANCE OF THE ADIABATIC CONDITION IN CALORIMETRY
}

BY FREDERICK BARRY

Received July 30, 1921

In recent communications to THIS JOURNAL a procedure was described by which the heats of slow reactions lasting 6 hours or more could be measured with precision. By an illustrative determination, the heat of inversion of sucrose by acid was fixed within an extreme experimental discrepancy of 4 gram-calories, which represented a variable error but little greater than that observed in the similar measurement of reactions complete in 10 minutes. This indicated that the precision of the protracted measurements was limited more by defects inherent in the general method than by imperfect control of the minute but slowly cumulative leakage which the procedure had been designed to minimize. There was, on the other hand, no evidence to show that such leakage had been prevented; indeed systematic errors were suspected, and the accuracy of the determinations was acknowledged to be in doubt. The present paper describes an attempt to discover the sources of both the variable and the constant errors which significantly affect measurements of this type, and thus to establish for them a workable standard of true accuracy. 'The attempt has been successful to this extent: that all thermal disturbances appear to be identified, which in protracted determinations 10 hours long are likely to cause errors of half a gram-calorie or more. The possibility of eliminating these effects (by means which the present work suggests) is now being tested by a more minute examination of the sucrose inversion. Meanwhile the results here presented possess a certain interest in themselves, since in many ways they are of general applicability.

\section{Thermal Disturbances Examined}

In nearly all precise adiabatic calorimetry heretofore, an open calorimeter has been used, surrounded, at least laterally and below, by very narrow air spaces, and stirred reciprocally. ${ }^{2}$ 'The thermal disturbances recognized as sources of probable error in the preceding research were for the most part characteristic of this type of system. They had been traced to the following sources: (1) direct conduction leakage between calorimeter and outer environment through thermometer, stirrer rods and operating mechanism, and through the insulations which enclosed these parts; (2) direct convection leakage between calcrimeter and outer environment, caused by the pumping of air by reciprocating stirrers; (3) complicated disturbances of leakage between calorimeter and bath, caused by the accidental presence of moisture in the insulating air gaps, and

1 This Journal, 42, 1295, 1911 (1920).

2 See ibid., 37, 994 (references) and 1006 (1915); ibid., 38, 1475 (1916); 39, 2113 (1917); 42, 1623 and 1914 (1920). But cf. Bur. Standards Bull., 11, $210 f f$. 
by various superimposed effects of evaporation from the open system; (4) imperfectly adiabatic initial conditions due to similar causes, themselves the result of diverse prior conditions; (5) various effects of transmission and distribution lags; (6) possible irregularities in the production of stirring heat; (7) thermometric uncertainties caused by environmental influences.

The present investigation comprised an examination of all these sources of uncertainty.

\section{Thermometry}

Temperatures were determined by the twin mercury thermometers previously used and elsewhere described. ${ }^{3}$ The reading error of these instruments was less than $0.0005^{\circ}$. The probable error of calibration, never greater than $0.0010^{\circ}$, was for the small temperature differences observed, which were read on selected ranges, considerably less than this." 'There was no evidence of significant capillary friction with changes more rapid than $0.002^{\circ}$ per hour, and the few irregular fluctuations of this magnitude which were observed were of ambiguous origin. ${ }^{5}$ In all cases where such irregularity was apt to cause significant error, the measurement was checked by change of procedure. The thermometers were installed and manipulated as formerly, so as to eliminate the danger of irregular registrations due to dissimilar or variable immersions and exposIures; and were further protected from the body heat of the operator by a mirror, and from the possible effects of illumination by water screens, which adequate tests showed to be completely effective. Thermometric lag introduced no errors. In every measurement the total thermometric error, thus made determinable and always estimated, was found to be too small to invalidate inferences drawn from the relations of the thermal effects measured, ${ }^{6}$

${ }^{3}$ Ref. 1, pp. 1918--1920.

4 The calibration had been made by comparison with normal instruments (made by Baudin and standardized by the Bureau des Poids et Mesures) in a carefully controlled adiabatic system. Sixteen points in three groups, each of which covered the total range of $0.6^{\circ}$, were thus determined, and checked each other with remarkabie nicety. In measurements of small changes of temperature, the ranges selected were on smooth slopes of the calibration curves close to chosen determined points. Larger temperature changes were measured over the complete segments between determined points. In this manner errors of interpolation were largely avoided.

"That is, possibly due to thermal inhomogeneity within the calorimeter, to abrupt irregularity of capillary bore, or to momentary change of stem temperature.

${ }^{6}$ In the measurements of the heats of stirring at lowest speeds (Tables I and II), of leakage rates due to conduction between calorimeter and outer environment (Table IV), of the final and vanishing rates of temperature change caused by surface adsorption of moisture (Table IX), and of certain thermal leakages between calorimeter and bath under heads of $0.1^{\circ}$ (Table V), the total temperature changes observed were less than $0.01^{\circ}$. In these cases, thermometric error caused uncertainties of measurement amounting to $10 \%$ or more. But all these data were either checked by repetition or were confirmed by their concordance with the results of more dependable measurement. In the succeeding tables, the error of observation for every recorded rate of temperature 
and the continued use of these instruments was thus for the time being justified. ${ }^{7}$

\section{Control of Outer Environmental Temperature}

The constant temperature closet previously built' and later considerably improved was used throughout. Since a very carefully controlled environmental temperature appears to be essential in protracted calorimetry (p. 916 and Table IV), this device merits a brief description. 'The closet was built of wood with double walls and floor in the middle of a cellar laboratory. Its walls were pierced near the ceiling with adjustable apertures, so that when the temperature outside it remained $2^{\circ}$ or more below that within, the latter could be made naturally to fall at a rate fairly definite and not too slow. By means of ice water flowing through a grid on the ceiling, this natural fall could be maintained also within the closet itself, so that it could be used in hot as well as in cold weather. Against this temperature fall an electrical heating and distributing mechanism operated, so as to establish a slightly fluctuating temperature. The control was maintained by a bimetallic regulator and relay arrangement, in all essentials similar to those commonly used with water thermostats. In principle, therefore, the room was a thermostat of this type.

'To make a fairly uniform distribution possible, the heating device was built on the outer wall of the closet. A small blower fan sucked air slowly upward through a vertical insulated box containing adjustable resistances, change may be computed by comparing the temperature changes observed (rates $X$ durations) with $0.0010^{\circ}$ (maximal thermometric error) or with $0.0005^{\circ}$ (the probable thermometric error).

T The data provided by this investigation give support to the opinion that excepting in rare instances, the calorimetric uncertainty-that due to the effects of natural thermal disturbances in imperfectly controlled systems-has been in the past considerably greater than the necessary thermometric error of mercury instruments. Such usually (cf. p. 934) has been the case whenever open calorimeters have been used, or whenever in determinations carried out with closed systems no particular precautions have been taken to avoid surface contaminations or the presence of water in the air gap. It is always likely to be the case whenever undetermined lags occur, and is inevitably so in all protracted determinations. If, in addition, the errors which are characteristic of particular physico-chemical processes are considered, it will be apparent that the use of mercury instruments will continue to be advantageous in all experimental work designed to establish the efficacy of special calorimetric procedures. When, however, these sources of uncertainty have been brought under definite control, a more exact electrical thermometry will certainly justify itself. The most troublesome defects of mercury thermometers, particularly those due to large and uncertain errors of calibration, to the recessity of stem exposure, and to capillary friction, may doubtless be considerably improved; and the possibility of such improvement merits consideration. There is no doubt, however, that the technique of the most precise mercury thermometry is already about as exacting as any other.

${ }^{8}$ Ref. 1, p. 1915. 
where it was heated to any desired temperature. In hot weather a grid of metal plates, through which ice water flowed continuously, replaced the coils. From this box the air was blown across the regulated heating amps, which were held in a horizontal metal box of somewhat smaller cross section, uninsulated and set out from the wall (so that it radiated ireely and was without great lag) and was delivered into the closet through a hopper shaped frame so placed that the incoming stream, if unchecked, would not impinge upon any operating mechanism. It was then caught between two horizontal and opposed air currents from swinging eight-inch lans, and was thus scattered. Smoke tests showed that the distribution thus effected was sufficiently rapid. The ventilation was, of course, excellent. The space $(4.0 \times 2.7 \times 2.7$ meters $)$ was sufficient for two calorimeters with all desirable accessories, and could be used by two operators without disagreeable crowding. ${ }^{9}$

The closet held necessary water in a single closed 1 jo-liter tank, fed from a similar tank above it on the outer side of the wall. In this tank water was heated uniformly and automatically to calorimeter temperature before it was introduced. Residual water in the inner tank was similarly brought to the same temperature before mesaurements were made. ${ }^{\text {in }}$

'The illumination of the closet was direct, from water-screened tungsten filament lamps, and the walls and large fixtures were painted black. This defined the sources of possible irregularities from radiation effects in all measurements. It is, however, probable that brighter indirect illumination in a white walled room would have been safe enough.

By these arrangements the temperature in the vicinity of the calorimeter was continuously held to within $0.2^{\circ}$ or even $0.1^{\circ}$ of the calorimetric. As will be shown later, this control was necessary but sufficient to reduce the correction for direct leakage between the calorimeter and its outer environment during 10 hours or less to magnitudes calculable without significant error from the determined leakage rates for large thermal heads (Table IV).

\section{New Modifications of Calorimeters}

The calorimeters used were two similar systems of the approximate dimensions and heat capacities most practical for general thermochemical work. ${ }^{11}$ They were built to accommodate without essential modification

${ }^{9} \mathrm{~A}$ closet formerly used $(3.6 \times 1.8 \times 2.4$ meters $)$ was not only inconvenient even for one operator, but was less easily regulated. In this construction, the air was taken from the closet itself instead of from the outside laboratory. Such construction eliminates the preheating and precooling boxes, but necessitates the installation of a ventilating fan. It is less fiexible than the present arrangement, but would serve well in case gases were set free within the room.

${ }^{10} \mathrm{It}$ is unnecessary to make a thermostat out of the inner tank.

${ }^{11}$ For the sake of possible future comparisons of data, the essential figures are here given: height of open cylindrical vessel, $152 \mathrm{~mm}$., of closed vessel, $154 \mathrm{~mm}$; diameter, 
any type of thermometric instrument, various inner vessels, electrical resistance, accessory thermo-elements and so on, and they were provided with several jackets by which they could be surrounded by air spaces of various widths and configurations, and with external mechanisms by convenient adjustment of which they could be stirred reciprocally at several speeds by one or two stirrers operating in conjunction or opposition, continuously or intermittently. A single cylindrical calorimetric vessel of gold-plated

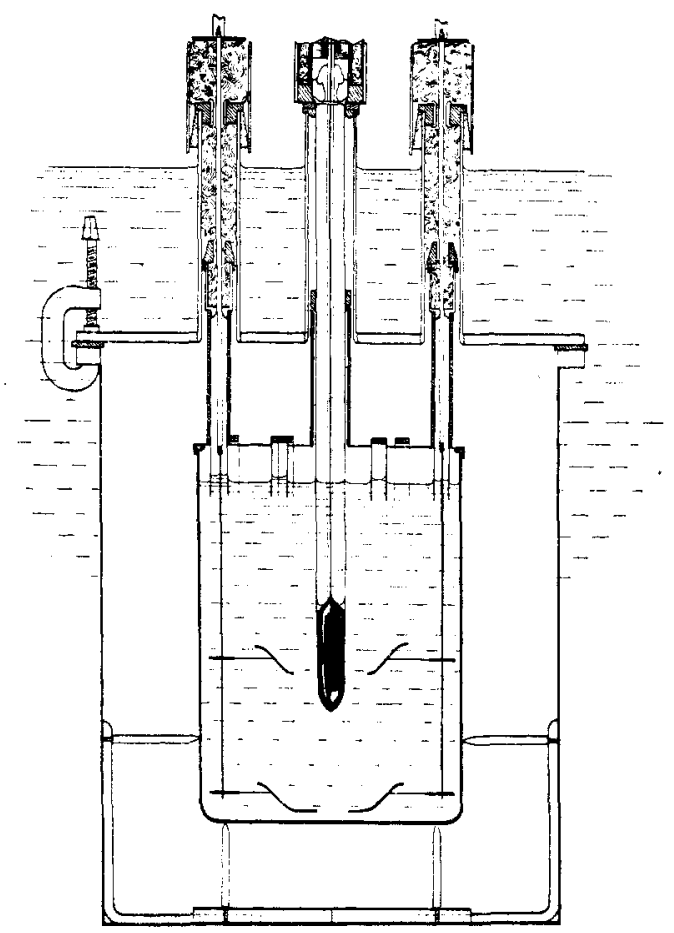

Fig. 1.-Closed calorimeter with $40 \mathrm{~mm}$, jacket. Metal is blank, or, as wire or sheet, is black; vulcanite is heavily and rubber lightly hatched; wax or kneaded rubber, cross hatched. Mercury, mirror surfaces and cement are black; ivory and glass are unmarked.

Parts behind the (diametrical) section are lightly drawn. The calorimeter support is represented as having 4 arms; in reality it has 3 . The stoppered apertures in the calorimeter cover are those for a second stirrer and electrical leads. They help to indicate the whole extent of metallic connection between calorimetric liquid and cover. Other accessory contrivances not used in this work are not drawn. Reduced to onequarter actual size.

$108 \mathrm{~mm}$; heat capacity here invariably used, 1234 gram-calorie units for both closed and open systems. 
copper was used throughout. This was fitted with a brass cover which bore vertical chimneys of vulcanite to accommodate thermometer and stirrer rods, and which, excepting at the stirrer apertures, could be made vapor-tight by gaskets of kneaded rubber. The cover was in thermal contact with the calorimeter water through inner brass extensions of the chimney collars as well as through the vessel walls. It was thus held closely enough at calorimeter temperature during the slow changes here measured.12 The calorimeter thus arranged is that designated in the sequel "the closed calorimeter." Without the cover it is called "the open calorimeter." In all other respects excepting for their insulations, the two systems were alike. In the determinations the vessels were filled with such definite weights of water that their total heat capacities were the same and invariable within the percentage error of measurement, and the same thermometer and stirrer were used with both.

The jackets were of the general type heretofore employed. Four of these jackets were used in this work, of such internal dimensions that when they held the closed calorimeter, the air gaps which surrounded it were uniformly 10, 20, 30, and $40 \mathrm{~mm}$. wide. The jacket rims were all made of the same external diameter, so as to fit a single cover, a plate of rolled brass into which chimneys corresponding to those on the calorimeter cover but larger, were tapped and soldered. Closure was effected against

12 For sealing the calorimeter, kneaded rubber was found preferable to wax, which by smearing was likely to affect the surface of the calorimeter and to contaminate its contents. The rim gasket here used, placed at the inner edge of the cover, suffices for measurements with water, but in chemical operations it should be placed outside as, for instance, between the edge of the cover rim and a ring firmly attached to the outer vessel wall a little below the top. A construction possibly safer in the measurement of more rapid changes would be that described by White [Phys. Rev., 25, 137 (1907); $31,574,671$ (1910) ]; a depression of the central cover surface which touches the surface of the contained liquid. This device, however, would restrict unfavorably the working space within the calorimeter. The use of oil seals with such construction would, of course, be inadvisable in chemical operations, and would justify itself in any case only if evaporation, the rate of which would be modified in an unpredictable way by slight surface contamination with oil, were wholly prevented. In adiabatic work, particularly in the measurement of slow processes, a space between the calorimeter liquid and a close fitting and highly conducting cover is not a disadvantage whenever the adiabatic condition can be closely maintained. Distillation between cover and liquid, slight even under very imperfect adiabatic conditions, amounts to nothing more than added internal distribution lag.

The vulcanite chimneys seem at first sight a more serious defect in construction. It is almost certain, however, that in adiabatic work, when the temperature of the bath fluctuates slightly about that of the calorimeter, they will have no appreciable lag, since their own heat capacity is a very small fraction of the whole, and since under these conditions they will transmit no measurable heat to and from the system. In many of the present measurements, in which fairly large thermal heads were established between calorimeter and bath, the chimneys must have introduced a considerable lag; the effect of this and of other lags was, however, eliminated by establishing in every measurement a uniform rate of flow under constant head, before readings were accepted for record. 
soft rubber gaskets by loose clamps, conveniently manipulated with a socket wrench. Within the jackets the calorimeter rested upon and was centered by pointed ivory rods, cut to the exact widths of the several desired gaps and screwed into sockets on a radically adjustable and rigid frame. This was secured in the looser jackets by graduated wedges with which it could be symmetrically adjusted. The dimensions of the jackets were, however, sufficiently uniform and exact to make the actual widths of the air gaps so close to multiples of $10 \mathrm{~mm}$. that they could be accepted as such without significant error. The jackets and jacket cover were nickel plated and highly polished inside, and the outer surfaces of the calorimeter were similarly, though not so perfectly, reflecting.

To prevent significant leakage through the stirrer apertures of the closed calorimeter, the following construction was adopted. The stirrer wires at a point which lay $10 \mathrm{~mm}$. above the liquid surface when the stirrer was in its lowest position, were tapped
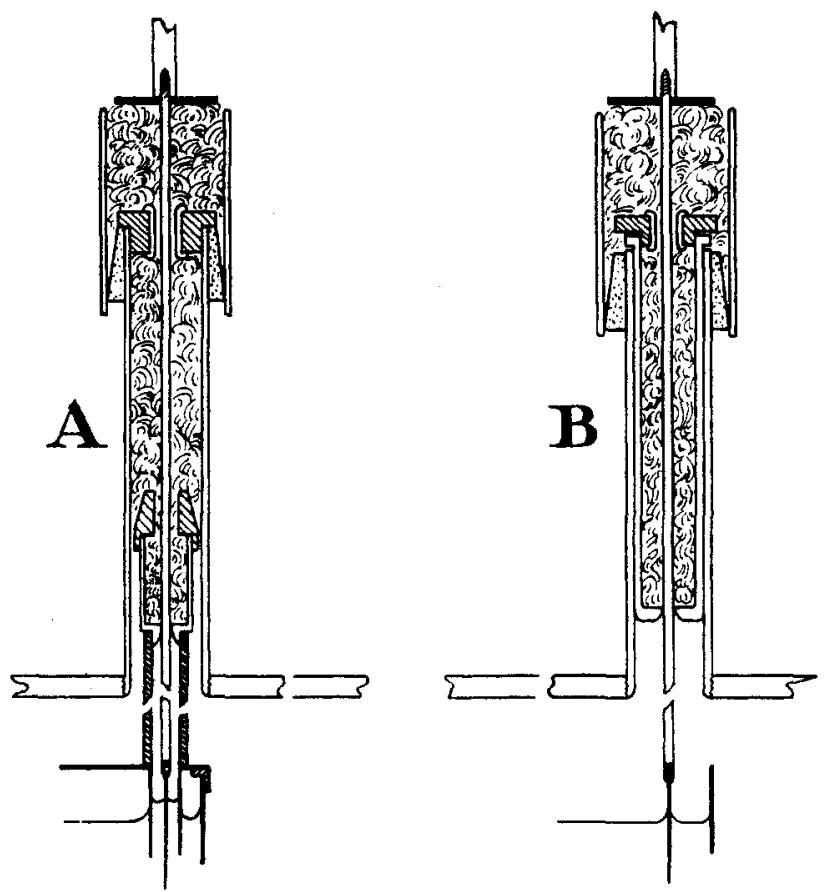

Fig. 2.-Detail of stirrer insulations: A, for closed calorimeter;

$B$, of open calorimeter. One-half actual size.

into polished rods of ivory less than $2 \mathrm{~mm}$. in diameter. Within the vessel these rods were wholly enclosed by those inner extensions of the narrow brass collars of the chimneys through which intimate thermal contact between cover and calorimeter liquid had been effected. Thence they extended through the vulcanite chimneys. . These were of such length that they traversed the widest upper basal gap and entered the corresponding chimneys of the jacket cover. They were surmounted by cylindrical brass baxes, pierced below with conical apertures just large enough to receive the rods, and filled with cotton wool firmly compressed and held in position by threaded vulcanite caps. This arrangement reduced the total volume of water-saturated air set in motion by the stirrers, and thus minimized convection in the chimneys, permitted no conduction of 
heat through stirrer wires into the insulation, and ensured a dry and compressed packing which held practically still air. All frictional heat due to scraping was developed in the boxes and was conducted through the brass into a narrow ring of air in close contact with the bath, so that it affected neither the calorimeter nor the insulating spaces. The narrow aperture of the box served also to steady the motion of the stirrer. Above the vulcanite chimneys, the jacket chimneys into which they extended were also stopped with cotton wool, held in place by perforated rubber stoppers lined with glass; and at the top were fitted with glass tubes similarly filled, which served primarily to prevent any contamination of the insulation beneath by spattering from the bath. ${ }^{13}$

The apparent efficacy of this form of closure for the covered calorimeter suggested the advisability of closing the air gap in a similar way when the open calorimeter was used. The usual stirrer chimney insulation heretofore employed-cotton wool held loosely or enclosed in glass tubes at the chimney tops-is very slightly effective on account of the large surfaces exposed to environmental temperatures, as will be shown in any case by rough calculations from the dimensions and thermal conductivities. In these measurements, therefore, the insulation was held in heavy brass cartridges, pierced with small apertures below, and closed above with perforated rubber stoppers lined with glass, which fitted snugly into the jacket stirrer chimneys throughout their entire length. $\mathrm{By}$ this device the stirrer rods from the open calorimeter were made to pass through long tubes of cotton wool insulation, the lateral areas of which were kept at bath temperature, so that conduction leakage through them was very small indeed. The efficacy of these insulations was demonstrated by the constancy with which the isothermal condition could be maintained (p.908); by the very slightly variable heating caused by fast continuous stirring of uniform speed in different assemblages (p. 909); by the small leakages between calorimeter and outer environment which resulted from conduction and convection through the stirrer orifices (Table IV and Ref. 28); and by the results of certain measurements (Table III) in which while slow continuous stirring was maintained, the leakage rates were identical under positive and negative bath heads as great as $1^{\circ}$.

In both constructions, the ivory rods extended into the outer air, where they were screwed into metal rods, which in turn were attached by nuts securely but loosely to the stirrer hanger. The operating mechanism was that employed before, modified by the inclusion of an extra countershaft and pulleys which permitted its adjustment to several speeds. The variability of these speeds was slight, and introduced after suitable correction according to former practice ${ }^{14}$ no appreciable irregularities into the calculation of stirring heats. The stirrer itself was a perforated 2 -stage ring provided with extra blades so disposed that with every stroke the calorimeter liquid was very thoroughly agitated. The thermal effects of stirring are discussed further on (p.909).

The bath was regulated by ice water and sulfuric acid drips as heretofore so that its temperature fluctuated about that of the calorimeter over a range of less than $\pm 0.01^{\circ}$. Correction was always made for leakage between calorimeter and bath on the assumption

${ }^{13}$ Other forms of closure were, of course, possible. The simple device of flexible rubber tubes attached to stirrer rods and chimney tops and certain practicable though more or less elaborate forms of liquid seal closures were, despite their probably greater efficiency, left untried because of the eddying air currents they were likely to set up in the air gaps.

In one test of the adiabatic insulation, the cotton wool in the boxes was saturated over its middle zone with heavy cylinder oil. The precaution was quite practicable, for no oil leaked out; but it proved to be unnecessary. Vaseline was too viscous to be used advantageously in this way.

1 From the data of Table I and according to the procedure of Ref. 1, p. 1936. 
previously made that the leakage was proportional to thermal head. The leakage measurements hereafter described (pp. 918, 934; Tables $\mathrm{V}$ and $\mathrm{X}$ ) proved that this assumption alone involved no significant error in measurements made either with the closed or with the open system. The bath was stirred by 2 multiple paddles, so effectively that having been locally warmed or cooled by the drips, it always came to complete thermal homogeneity within 50 seconds. This delay was the same for rise and fall of temperature. and introduced compensating errors into the record of its slight and regular fluctuation which calculations from the minute data of former protracted measurements proved to be quite negligible. It was probable that the thermal inhomogeneity of the bath was too small to affect sensibly the uniform distribution of heat over the highly conducting jackets: these, therefore, were assumed to be at every instant during measurement practically isothermal. ${ }^{15}$ Convection lag within the jackets was certainly quite negligible. ${ }^{16}$ The volume of the largest air gap was 4.8 liters. Its heat capacity at the temperature of measurement, 1.4 gram-calorie units, was too small to affect the results of usual measurement even if it were wholly neglected (Ref. 11) and in any case its effective heat capacity could be estimated with negligible error. Calculation from its determined thermal conductance (Table VIJI) also showed that under the adopted bath control its conduction lag would be too small to affect with significant error the calculation of the thermal transfer between calorimeter and bath, which ignored this effect. ${ }^{17}$ It is therefore apparent that neither the heat capacity nor the total thermal lag of the widest air gap could have any perceptible effect on the results of protracted measurements made with systems similar to those here employed; and that, finally, the procedure formerly adopted for the reduction and correction for leakage between calorimeter and bath involved no significant error. This correction was never greater than $0.0005^{\circ}$; and when the wider gaps were used, was wholly negligible.

${ }_{15}$ The convection leakages under very small heads suggested, however, that they may not have been so (Ref. 37).

${ }_{16}$ There was no need to attempt the difficult determination of such lag. Under an adiabatic control even carelessly maintained, the temperature difference between calorimeter and bath will never be greater than $0.1^{\circ}$. With a 10 - or a $20 \mathrm{~mm}$. air gap, the total convection leakage under this head is not more than $0.013 \mathrm{~g}$.-cal. per minute, and with the $40 \mathrm{~mm}$. gap it is about half as much (Table VI and Ref. 11). Even if this leakage were wholly prevented by lag, the resulting effect during periods of mixture, which when properly conducted are not longer than 5 minutes, and upon correspondingly large bath fluctuations of similar duration due to accident, would be negligible. The analogous effect on the leakage into a bath held continuously at a mean temperature $0.01^{\circ}$ lower than the calorimeter would be, if the narrowest $(10 \mathrm{~mm}$.) gap were used, less than $0.0013 \mathrm{~g}$.-cal. per minute, or $0.8 \mathrm{cal}$. in 10 hours, but in practice the bath fluctuates about the calorimeter temperature within a range of less than $\pm 0.01^{\circ}$ and the effect is, again, negligible.

17 The maximum bath head was $0.01^{\circ}$; the mean head of the insulating air, therefore, averaged $0.005^{\circ}$, and its average excess or deficiency of heat while leakage was constant under the maximal bath head was $1.4 \times 0.005=0.007 \mathrm{cal}$. Since the thermal conductance under $0.01^{\circ}$ head was 0.010 cal. per minute (Table VIII and Ref. 11) this heat at the maximum head would be delivered in $0.007 \div 0.010=0.7$ minute. It wotld therefore be in considerable part delivered during the reversal of the bath. Assuming such reversals to occur not oftener than every 10 minutes (the usual practice) or 60 times in 10 hours, the total heat retained by the gap from leakage in one direction would average less than $60 \times 0.007=0.4 \mathrm{cal}$.- -a quantity itself negligible, amounting to no more than $1 / 5$ of this leakage, and largely, if not wholly, compensated by the similar effect on leakage in the opposite direction. 


\section{Establishment of the Isothermal Condition}

When the closed calorimeter equipped with thermometer and stirrer was held in a dry air ${ }^{18}$ space with the bath kept fluctuating within $\pm 0.01^{\circ}$ and the outer environment within $\pm 0.2^{\circ}$ of the temperature within, it could be and was repeatedly maintained isothermal for 3 hours or more within the range of thermometric sensitivity. The duration of these tests and their variety with respect to absolute temperature and prior condition made it very improbable that this apparent constancy was due to anything but a real equilibrium. ${ }^{19}$

With the closed calorimeter in a dry gap, this condition was always casily established. With the open calorimeter, on the contrary, great difficulty was encountered, due not only to evaporation leakages, which could be prevented by saturating the gap with water, but to various protracted thermal disturbances caused by the surface adsorption of moisture and the occasional isothermal distillation from droplets which accompanied readjustments from diverse prior conditions (p. 934. Cf. p. 931). These effects could be most certainly eliminated by enclosing the open vessel in a dry gap and allowing it to rest at constant temperature for several hours (conveniently overnight) until equilibrium was established, a procedure which necessitated a thermostatic regulation of the bath. Such regulation could be avoided only by bringing the bath before measurement to the desired temperature, and holding it there while the jacket surfaces and that of the calorimeter, previously heated to a temperature somewhat higher, were slightly wetted and allowed to dry until the adhering water was almost invisible. Then, after quick assemblage, it was not impossible that equilibrium could be established within an hour, but this was by no means certain. In the long run, time would be saved by the former procedure. ${ }^{20}$ In either way, however, an isothermal condition could be established with the open calorimeter in a saturated gap, as exact as that effected with the closed calorimeter in a dry gap. Both systems, at rest under the habitual controls of bath and environmental temperature, functioned for 3 hours,

${ }^{18}$ In this paper the phrase $d r y$ air will mean not water-free air, but air so far unsaturated with moisture that a temperature fall of calorimetric range will not cause precipitation or supersaturation.

${ }_{19}$ The constancy of temperature was not disturbed by slow stirring which developed no measurable heat but which nevertheless was sufficient to maintain thermal homogeneity; and the occasional warming and cooling of the thermometer stem, which caused temporary displacements, after which the temperature of equilibrium was approached by rising and falling threads, resulted in no permanent change of reading.

${ }^{20}$ It was possible to establish the isothermal condition very quickly by dampening the calorimeter surface, assembling in a dry gap and introducing water into the gap afterward; but this procedure was calorimetrically impracticable, since with subsequent change of temperature, the presence of free water in the gap was a source of thermal disturbance. (P. 935.) 
and hence presumably for longer times, as thermostats precise to $\pm 0.0005^{\circ}$. Their behavior under fixed conditions of thermal leakage and under adiabatic change of temperature will be discussed later (pp. 918, 934).

\section{Heat of Stirring and Stirring Lag}

A. large number of measurements were made of the heat developed by reciprocating stirring, on account of the very dangerous uncertainties which may result in precise measurement from its incomplete control. Each test was made with the closed calorimeter, which previously had been held isothermal for an hour or more. In all cases the jacket was regulated with such care that the correction for leakage was negligible. Each test lasted from 40 minutes to 2 hours, and none involved variations in stirring speed large enough to make errors in the correction for this irregularity markedly significant. Since all temperature changes observed were slow, it was possible to read the thermometers closer than the usual $0.000 .5^{\circ}$, and such readings, marked + and - , were taken at 10 -minute intervals. The temperature ranges measured were chosen so as to avoid calibration corrections, and check measurements were made at different temperatures. The adequacy of these precautions was not assumed, but was proved in every case by the evaluation of all corrections.

The constancy of the rate of heat production under continuous stirring at identical speeds was first tested by measurements made with different assemblages, scattered throughout the whole series of experiments and over various ranges within a total compass of about $0.2^{\circ}$. It was found that continuous stirring at the constant rate of 56.0 strokes per minute during 30 minutes caused within reading error the same rise of temperature. Since this rate, maintained for this time, will be sufficient in all determinations of heats of reaction in solution to ensure a thorough and rapid mixing, it was thus demonstrated that with systems no more carefully constructed than those here used, mechanical irregularity caused by varying frictional heats developed within the systems would usually have an insignificant effect on the heat of stirring.

The variation of the heat of continuous stirring with change of speed was determined in three systems of widely different internal configurations. The results are given in Table $I$. The system there marked Calorimeter $A$ was that used in the present investigation (See Fig. 1); Calorimeter $S$ was a closed thermochemical system closely similar to that used in the determination of the heat of inversion of cane sugar by acid. ${ }^{21}$ Calorimeter $R$ was one which held between two reciprocating stirrers an electrical resistance enclosed in a loose vertical coil of small metal tubing. In the first two systems the stirring was performed by one stirrer; in the third

$"$ Ref. 1, Fig. 1. 
TABLE I

Variation of the Heat of Continuous Reciprocating StirRing with Speed

Rate of

change in

Expt. Duration Speed temperature the relation;

$$
R \sim S^{x}
$$

Influence on $x$

of probable

errors of measurement ${ }^{b}$ Min. per minute minute $\times 10^{6}$

$$
R
$$

$$
R
$$

Error of tem- Frror of stir-

perature de- ring rate de- Sum $c$

termination termination (maxima)

$$
\text { Calorimeter A: Temp. range }=.058^{\circ}
$$

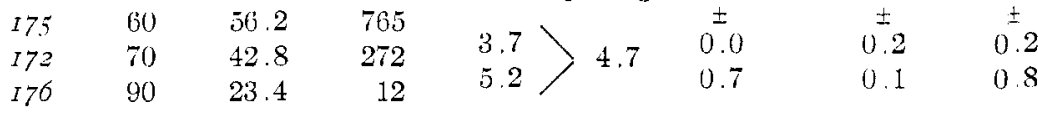

\begin{tabular}{|c|c|c|c|c|c|c|}
\hline 44 & 56.8 & 532 & & & & \\
\hline 60 & 45.7 & 332 & 3.1 & 0.1 & 0.0 & 0.1 \\
\hline 60 & 36.5 & $138^{a}$ & & 0.2 & 0.2 & 0.4 \\
\hline
\end{tabular}

CALORIMETER S, Series I: Temp. range $=.036^{\circ}$

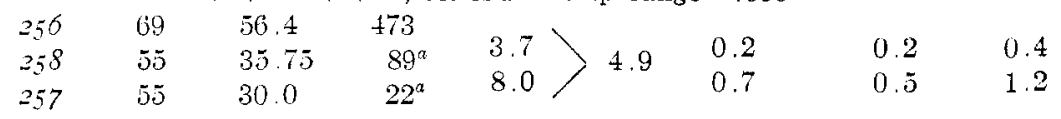

CALORIMETER S, Series II: Temp range $=.032^{\circ}$

CALORIMETER R: Temp. range $=.035^{\circ}$

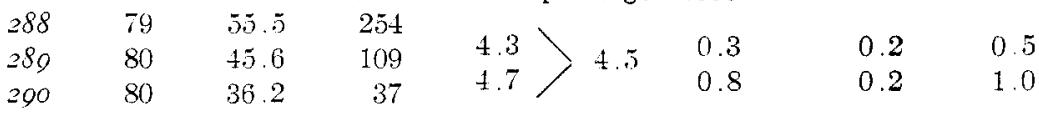

"Maximal values.

"Calculated for maximal influence, as affecting the smaller value in each comparison.

c The total error is probably less than twice this sum. Lateral displacements of the stirrer were slight, the length of stroke was invariable within $1 \mathrm{~mm}$. (in $50 \mathrm{~mm}$.), and the temperature range was too small to involve significant changes in viscosity, in every series. Certain duplicate measurements indicated that this estimate is too large.

${ }^{d}$ The omitted experiments in each continuous series were tests of the maintenance of the isothermal condition.

- The discrepancies between the rates of temperature change at corresponding speeds were due to differences in heat capacity, length of stroke, and mean temperatures. as well as to differences in the internal configurations.

by two stirrers in opposition. The heat developed in the first two systems was about the same; it was considerably less in the third system, in which more eddying occurred. Each group of measurements was made at one time with the same assemblage. It is evident from the data that at low speeds in all cases, the heat developed varies as the fourth and higher powers of the speed; and that with the two simpler systems certainly, and probably with the third, as the rate decreases, the heat developed falls off more rapidly. This variability is shown to be real by the calculated values in the last column of Table I, which exhibit the influences of the maximal probable errors of observation upon the determined index of variability; for if these errors be applied as corrections in the most unfavorable sense the magnitudes of the indices and their quanti- 
tative relations are not greatly disturbed. The sense of the change in variability, moreover, is supported by the results of former observation according to which, at higher rates, the heat developed varies as the cube and higher powers of the speed. ${ }^{22}$ The data further show that differences in internal configuration may have an effect upon these relations which in precise measurement cannot be safely ignored. The extraordinary sensitivity of heat production to change of speed here shown emphasizes also the need of maintaining with synchronous motors and gear transmissions or similarly effective controls, a measurably uniform speed in all continuous stirring; or else of observing the stirring speed at frequent intervals during measurement and of correcting for its variation. Firally the results prove that at speeds no greater than 12 strokes a minute in systems similar to those here employed (in which friction is comparatively large), the heat developed in 10 hours will be inappreciable by mercury thermometry. Since this rate will be sufficient to maintain thermal l.omogeneity in all reacting media not distinctly heterogeneous and rapidly changing, it is therefore evident that effective continuous stirring is permissible, even in protracted calorimetry.

In the preceding thermochemical determinations, which necessitated rapid continuous stirring during an initial period of mixture, and which vet demanded the development of very little stirring heat altogether, the reacting system, after solution was complete, was stirred intermittently at the initial rate by groups of strokes applied at 10 -minute intervals. 'This method would be advantageous in many cases, especially when stirring at different speeds would be inconvenient; and similar intermittent stirring at a slower rate would always be preferable to continuous stirring when very small temperature changes were measured, or in very protracted determinations. It had been shown ${ }^{23}$ that in homogeneous reacting liquids under adiabatic conditions such stirring, even when very slight, was sufficient to maintain a uniform calorimeter temperature during the progress of any slow reaction; and it was believed that, in the determinations referred to, the slight heat developed by this stirring was estimated with sufficient precision by assuming that the heat per stroke developed by continuous and intermittent stirring at the same rate was the same. This assumption, which left out of account the initial and final effects of liquid inertia, required confirmation. With the two calorimetric systems $A$ and $S$ (p. 909) it had been observed that after stirring at the maximal rate of 56.0 strokes per minute, noticeable motions of the liquid persisted for more than 2 minutes after stirring ceased. ${ }^{2-4}$ The procedure

${ }^{22}$ White, This Journal, 40, 1880 (1918). Richards and Burgess, ibid., 32, 448 449 (1910).

${ }^{23}$ Ref. 1, p. 1934.

${ }_{24}$ These motions were made apparent in a duplicate system which held a dilute acid-phenolphthalein solution in glass, by the movements of filaments of alkaline solu- 
of the tests was suggested by this observation. Three series of measurements were carried out. In the first of these the closed calorimeter was used in a wide air gap, and was left unsealed, so that a slow evaporation took place which, with minimal stirring under the customary controls of bath and environment, caused a measurably constant fall of temperature. Against this fall the rise due to heat of stirring was developed. The stirring speed was constant, and the stirring was both continuous and intermittent, a definite number of strokes being applied at 5 -minute intervals in measurements of the latter sort. The measurements were of varying duration and were made and repeated in irregular succession. In the second series this procedure was repeated with the sealed calorimeter, which, with minimal stirring under the same conditions, remained at constant temperature. It is clear that in these two series, the same rates of temperature change-in particular the minimal rates, which were most liable to error-were developed by different rates of stirring, and the experimental uncertainties, consequently, greatly reduced by a comparison of results. A further check upon the precision of measurement was provided by the practice of stirring by groups of strokes of varying duration at equal intervals, since in a given time all such stirrings involved the same end effects but caused different rates of temperature change. The two sets of data (Series I and II of Table II) are closely consistent, and prove that within the thermometric reading error, and for 10 to 20

TABLE IT

Heats Developed by Continuous and Intermittext Stirking at the Same Speed

\begin{tabular}{|c|c|c|c|c|c|c|c|c|c|c|}
\hline 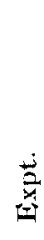 & مَّ & 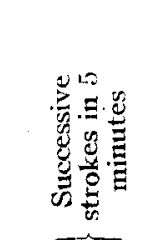 & 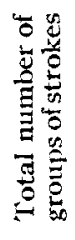 & & 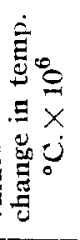 & & 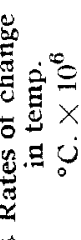 & $\stackrel{\Xi}{0}$ & 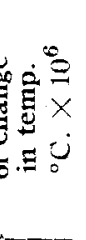 & 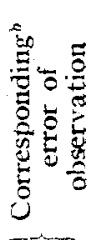 \\
\hline M & & & & $\begin{array}{c}\text { Per } \\
\text { min. }\end{array}$ & $\begin{array}{c}\text { Per } \\
\text { stroke }\end{array}$ & $\begin{array}{c}\text { Per } \\
\text { min. }\end{array}$ & $\begin{array}{c}\text { Per } \\
\text { stroke }\end{array}$ & $\begin{array}{l}\text { Per } \\
\text { min. }\end{array}$ & $\begin{array}{c}\text { Per } \\
\text { stroke }\end{array}$ & \\
\hline & & & & & RIES I & & & & & \\
\hline 87 & 45 & $\begin{array}{c}285 \\
\text { (Continuous) }\end{array}$ & . & 578 & 10.1 & 578 & & \pm 0 & \pm 0 & \\
\hline 88 & 50 & 60 & 10 & 126 & 10.5 & 124 & & +2 & +0.4 & +2 \\
\hline$Q I$ & 50 & 60 & 10 & 105 & 8.8 & 124 & 10.1 & -9 & -1.3 & -7 \\
\hline 89 & 60 & 30 & 12 & 65 & 10.8 & 62 & & +3 & +0.7 & +2 \\
\hline po & 60 & 15 & 12 & 33 & 10.9 & 31 & & +2 & +0.8 & +1 \\
\hline 93 & 45 & 15 & 9 & 35 & 11.7 & 31 & & +4 & +1.6 & +2 \\
\hline
\end{tabular}

tion, which were produced when a drop of sodium hydroxide solution was placed, directly after stirring had ceased, on the surface of the acid. An immersed centimeter scale exhibited the minimal rates of liquid motion. This simple device served equally well to show the directions of the more rapid currents and their relative rates of retardation in different parts of the system. 


\begin{tabular}{|c|c|c|c|c|c|c|c|c|c|c|}
\hline \multicolumn{11}{|c|}{ SERIES II } \\
\hline 1.42 & 65 & $\begin{array}{c}280 \\
\text { (Continuous) }\end{array}$ & $\cdots$ & 687 & 12.3 & 686 & s & -1 & \pm 0 & \pm 0 \\
\hline 1.75 & 65 & $\begin{array}{c}280 \\
\text { (Continuous) }\end{array}$ & $\ldots$ & 686 & 12.3 & 686 & & \pm 0 & $\neq 0$ & \\
\hline 179 & 60 & 60 & 12 & 133 & 11.5 & 147 & 12.3 & -14 & -8 & \pm 13 \\
\hline 1.43 & 75 & 30 & 15 & 60 & 10.0 & 74 & & -14 & -2.3 & -7 \\
\hline 1.76 & 100 & 30 & 20 & 76 & 12.2 & 74 & & +2 & +0.1 & \pm 0 \\
\hline 1.47 & 75 & 15 & 15 & 37 & 12.2 & 37 & & \pm 0 & +0.1 & \pm 0 \\
\hline 1.48 & 120 & 8 & 25 & 12 & 7.3 & 20 & & -9 & -5.0 & -10 \\
\hline \multicolumn{11}{|c|}{ SERIES III } \\
\hline 203 & 50 & $\begin{array}{c}280 \\
\text { (Continuous) }\end{array}$ & . & 580 & 10.4 & 581 & \multirow{4}{*}{10.4} & -1 & \pm 0 & \pm 0 \\
\hline 2,06 & 50 & $\begin{array}{c}280 \\
\text { (Continuous) }\end{array}$ & $\ldots$ & 582 & 10.4 & 581 & & +1 & \pm 0 & \pm 0 \\
\hline 204 & 180 & $\begin{array}{c}16.7 \\
(10 \mathrm{in} 3 \mathrm{~min})\end{array}$ & 60 & 40 & 12.0 & 35 & & +5 & +1.6 & +4 \\
\hline 207 & 200 & $\begin{array}{c}16.7 \\
(10 \mathrm{in} 3 \mathrm{~min})\end{array}$ & 67 & 37 & 11.1 & 35 & & +2 & +0.7 & +4 \\
\hline
\end{tabular}

"For normal values, parts of the rates of change for continuous stirring are taken, proportional in every case to the relative numbers of strokes applied in unit time (5 minutes). The divergence of the experimental and normal values thus measures the divergence of the determined heats of intermittent stirring from strict proportionality to the corresponding heats of continuous stirring.

b The error values here represent in each case the temperature difference which would, under the conditions of determination, occasion the divergence observed. They were calculated from the extended data of measurement, which are merely summarized here.

'Determinations 92 and 144 of the continuous series were interpolated tests of lcakage constancy and isothermal condition respectively.

The measurements of all series were made in the succession indicated by their expt. numbers: those of Series I on one day; those of Series II and III on two successive days. The differences between corresponding absolute values in the three series is due to slight differences of construction in the systems used, to constant error in the estimation of evaporation leakage in Series I, and to the omission of certain constant corrections in Series I and II. Since Series II involved two assemblages, it is clear that no error entered as a result of mere readjustment of the system.

The measurements most affected by capillary friction were those of Expts. 89 and 148 . It will be noted that these exhibit no unusual divergence from the normal. The only divergence which suggests that liquid inertia has a measurable effect is that of Expt. 149: but the correspondingly large negative divergences of Expts. 91 and 143 deprive this result of any significance.

groups of strokes, the average heat per stroke developed by intermittent and continuous stirring is the same. 'The thermometric precision during slow changes of temperature having been established by these preliminary experiments, two determinative tests were finally made, each of 3 hours' duration (Series III of Table II), in which groups of 10 strokes were applied at 3-minute intervals. These yielded a heat per stroke so close to that determined for continuous stirring as to ensure the absence of sig- 
nificant error in correcting for the heat of intermittent stirring developed according to former practice in 10 hours, on the basis of the original assumption. ${ }^{25}$ It was thus demonstrated that in protracted adiabatic measurements with homogeneous systems, intermittent stirring is permissible as a valuable alternative practice, advantageous whenever it is desirable to reduce the stirring heat to the least possible magnitude.

Under adiabatic conditions, a minimal effective intermittent stirring can always be determined by trial readings of temperature taken directly before and directly after the stirring is applied. Under merodiabatic conditions, such tests always reveal a considerable lag even when the thermal head is small. This lag, which is obviously occasioned by temperature differences between the interior and the periphery of the calorimeter established by leakage during the intervals of quiescence, is a source of possibly dangerous error in measurement, since because of it the established head is variable and its mean value always less than that observed. It seemed desirable to determine a limit of toleration for possible irregularities of behavior thus occasioned when in adiabatic practice accidental bath heads are established through temporary loss of control. 'The closed calorimeter surrounded by air gaps 10,20 , and $40 \mathrm{~mm}$. wide was subjected under each of these conditions to bath heads of $0.6^{\circ}$ and $1.00^{\circ}$ both higher and lower, which were consistently maintained by the bath drips constant within the same limits of precision as those of adiabatic measurement. ${ }^{26}$

The experimental procedure was identical with that of the leakage measurements, which is fully described elsewhere (p. 920) but it was probably more exact, for the thermal effects measured were so small that excessive precautions had to be taken to determine even the sense of their variability. Under each condition of measurement the thermal leakage

${ }^{20}$ This proportionality will obtain only when the stirring is sufficiently thorough. In parallel tests with other systems, it was found that when any large volume of liquid was left untraversed by the stirrer the normal flow was not established even by 20 strokes of vigorous stirring. This was shown by the initial rates of temperature change, which in one case were less than normal for over 2 minutes, although in this time a thorough mixing was accomplished (Ref. 24). In these cases the heat per stroke in intermittent stirring may be very much less than that of continuons stirring at the same rate. This is advantageous, of course, if the heat developed is negligible: otherwise with every system used the conditions of proportionality should be established by trial.

${ }^{25} \mathrm{It}$ seems probable that, when by proper means convection leakage is eliminated, highly accurate determinations may be made under a condition of constant leakage thus maintained. All of the leakage measurements here described are instances of such determination. The advantageous applicability of this condition to other than strictly calorimetric measurement, to the measurement of the thermal conductivity of gases and other thermal insulators, for instance, is obvious (cf. Ref. 41, third paragraph). It provides also promising opportunities for the development of precise and adequate procedures in isothermal calorimetry, which are at present under investigation in this laboratory. 
was determined; first, when the stirring was continuous at a speed which was found to be completely effective in maintaining thermal homogeneity at all times (about 24 strokes a minute) ${ }^{27}$ and second, when stirring was intermittent and slight, at the rate formerly used in thermochemical measurement ( 10 strokes every 10 minutes at a speed of about 56 strokes a minute). The results are given in Table III. The data show that the

TABLE III

\section{EFFECTS OF THERMAL INHOMOGENEITY UNDER INTERMITTENT StTIRRING ON CALORIMETER LEAKAGE}

\begin{tabular}{|c|c|c|c|c|c|c|c|c|}
\hline & & & & $\begin{array}{r}\text { Lea } \\
\text { egrees } p\end{array}$ & $\begin{array}{l}\text { me } \\
\text { min. } \times\end{array}$ & $10^{5}$ & & \\
\hline$\frac{\dot{0}}{2}$ & 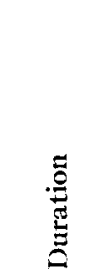 & 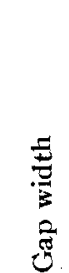 & 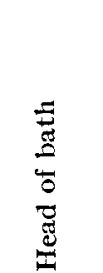 & 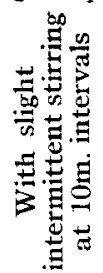 & 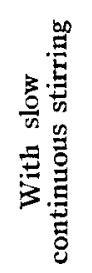 & & 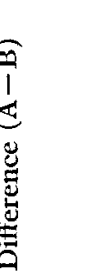 & \\
\hline & Min. & $\mathrm{Mm}$. & ${ }^{\circ} \mathrm{C}$. & $\underbrace{}_{A}$ & $\mathrm{~B}$ & $\begin{array}{c}\text { Degrees } \\
\text { per } \\
\text { minute }\end{array}$ & $\begin{array}{c}\text { Per } \\
\text { cent. } \\
\text { of B }\end{array}$ & \\
\hline 265,266 & 50,40 & 10 & -1.00 & -271 & -282 & 11 & 4.0 & \\
\hline $270,27 I$ & 45,41 & 10 & -1.00 & -276 & -283 & 7 & 2.5 & \\
\hline 267,268 & 40,62 & 10 & -0.60 & -160 & -178 & 18 & 10.7 & 8 \\
\hline 273,272 & 40,40 & 10 & -0.60 & -164 & -175 & 11 & 6.5 & \\
\hline 276,277 & 60,40 & 20 & -1.00 & -169 & -180 & 11 & 6.3 & \\
\hline 281,280 & 85,60 & 20 & +1.00 & +170 & +179 & 9 & 5.1 & \\
\hline 285,284 & 50,40 & 20 & -0.60 & -102 & -119 & 17 & $14.2^{a}$ & 8 \\
\hline 282,283 & 52,50 & 20 & +0.60 & +99 & +101 & 2 & $2.0^{a}$ & \\
\hline 226,225 & 61,70 & 40 & -1.00 & -170 & -183 & 13 & 7.1 & \\
\hline 234,235 & 50,50 & 40 & -1.00 & -165 & -186 & 21 & 11.3 & 0 \\
\hline 245,246 & 61,80 & 40 & -1.00 & -159 & -177 & 18 & 10.1 & \\
\hline 238,237 & 60,80 & 40 & -0.60 & -91 & -96 & 5 & 5.2 & \\
\hline 248,247 & 50,70 & 40 & -0.60 & -89 & -100 & 10 & 10.0 & 8 \\
\hline 254,253 & 61,56 & 40 & +0.60 & +86 & +96 & 10 & 9.6 & \\
\hline
\end{tabular}

"Discrepancy traced to faulty insulation: the errot is greater in the measurement under negative head, and the average is probably too low.

${ }^{b}$ The percentage corrections are taken in the probable ratio least favorable to an accentuation of the reverse curvatures of the leakage curves (cf. Table V).

thermal effect of the inhomogeneity under minimal intermittent stirring with the $40 \mathrm{~mm}$. gap system was proportional to the thermal head, but that with the 20 - and $10 \mathrm{~mm}$. systems, it increased somewhat less rapidly than the head. The maximal and minimal effects were such as to cause respectively

27 With the system here used held under a constant bath head of $1^{\circ}$, the measured rates of temperature change [corrected for a heat of stirring as determined under adiabatic conditions (Table I)] were identical when the rates of continuous stirring were 15 and 24 strokes per minute. 
about $8 \%$ and $4 \%$ diminutions of the leakages, which would imply about the same percentage diminutions of the actual thermal heads. The effects of measurement error in these determinations is indicated in the table: although relatively large, they seem not to affect the validity of the above inferences, and the systematic errors involved are in comparison quite negligible. It thus appears that the inhomogeneity permitted by intermittent stirring will introduce no error into adiabatic measurement even if accidental heads of $0.1^{\circ}$ are continuously maintained for 20 minutes or more (cf. Table III) and that it may be adequately though laboriously corrected for, if for any practical reason merodiabatic systems under heads of less than $1^{\circ}$ are intermittently stirred. It is obvious, however, that in properly conducted merodiabatic measurement the stirring should always be continuous.

Summarizing the practical results of these stirring experiments, it appears that in protracted adiabatic measurement, slow continuous stirring and intermittent stirring at any convenient rate are equally permissible. Intermittent stirring possesses the obvious advantage of developing less heat, and consequently of introducing into measurement smaller errors due to change of speed. It also diminishes leakage between the calorimeter and the outer environment when reciprocating stirrers are used, and is more convenient in measurements which necessitate a rapid transition from vigorous to slight stirring. With continuous stirring maintained at like speed for the same time these advantages are secured only by synchronous regulation of motor speed and faultless transmission, or by more frequent observations of change in the rate of stirring and a more minute correction for its variability, by more careful insulation and correction for leakage to and from the outer environment, and by the use of devices for quick readjustments of stirring speed, such as stepped pulleys or gear clutches. Intermittent stirring is therefore safer, excepting when elaborate and smoothly working mechanisms are available for speed control and, when stirring is reciprocating, for the regulation of outer environmental temperatures; or unless with continuous stirring a lower speed is effective. Experience suggests that, all things considered, a continuous stirring under precise control is preferable for exact protracted adiabatic measurement, unless the reaction period exceeds 10 or 12 hours, or unless the total change of temperature is very small. Under the latter conditions, slow intermittent stirring, which may be so conducted that negligible heat is developed even in very protracted measurement, appears to be advisable.

\section{Direct Leakage between Calorimeter and Outer Environment}

By approximate calculation from the known dimensions and thermal conductivities of those parts of the present system which passed directly 
from the calorimeter to the outside air, it was estimated that its leakage into an outer environment $1^{\circ}$ higher would be about 0.30 gram-calorie per hour, corresponding to a temperature change of $0.00023^{\circ}$ per hour, per degree thermal head. This leakage, if not corrected for, would cause in a measurement of 10 hours' duration, conducted at a mean environmental remperature $1^{\circ}$ higher or lower than the calorimetric, a constant error of $0.002^{\circ}$. It seemed desirable, therefore, to check the none too dependable estimate of its value by actual determination.

Since it was intended always to eliminate this leakage, rather than to correct for it, its maximal value alone was sought; and for this reason the less effectively insulated open calorimeter was used for the tests. The system was first brought to equilibrium close to $20^{\circ}$ under the usual controls, and the heat of continuous stirring at the maximal rate of 56 strokes

TARLE IV

DIRECT LEAKAGES FROM CALORIMETER TO FNVIRONMENT

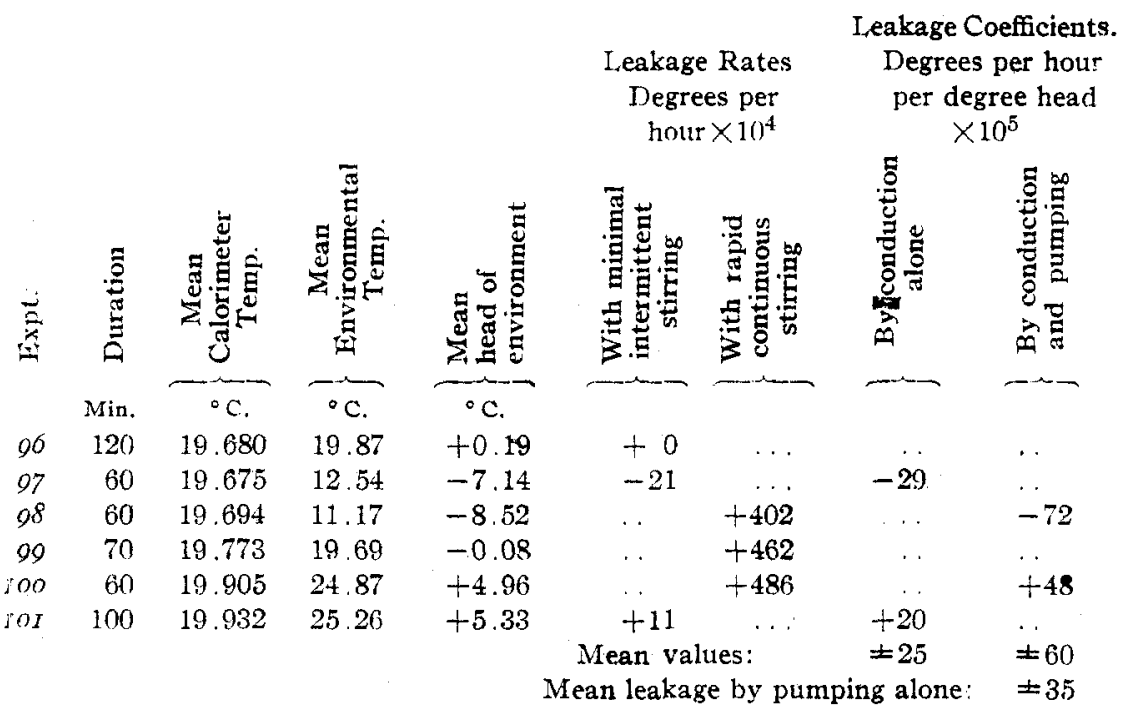

The leakage coefficients for conduction are consistent within measurement error $(<\neq 0.0005$ ). Those for pumping are not so. The latter effect seems to be accelerated with increase of thermal head, as would be expected.

per minute determined. The temperature of the surrounding air was then brought down to $11^{\circ}$ and the rate of temperature change within the calorimeter was measured while it was stirred, first very slightly and intermittently and then continuously at the maximal rate. The whole procedure was then repeated with the surrounding air at $26^{\circ}$. The data so obtained, corrected for all significant thermal disturbances, are shown in Table IV. They confirmed in a striking (and probably fortuitous) manner the results of previous' calculation, and showed also that the environmental 
control established (p. 902) was necessary but sufficient to prevent, in measurements made in the determined manner, the introduction of constant error either by conduction leakage or by pumping of air from calorimeter to outer environment. ${ }^{28}$

\section{Leakage between Closed Calorimeter and Bath}

The most instructive of the present measurements were those of thermal leakage through the insulating air spaces between calorimeter and bath. Among them, those least affected by thermal disturbance were measurements made with the closed calorimeter in water-free gaps. The carefully sealed vessel was enclosed successively in dry air spaces 10, 20 and 40

TABLE V

LEAKage BeTweEn Closed CALORIMETER ANd Bath

SERIES I

All Leakage Values in Degrees per Minute $\times 10^{5}$

\begin{tabular}{|c|c|c|c|c|c|c|c|}
\hline $\begin{array}{l}\text { Gap } \\
\text { Width } \\
\text { Mm. }\end{array}$ & $\begin{array}{c}\text { Thermal } \\
\text { Head } \\
\text { of bath } \\
{ }^{\circ} \mathrm{C} \text {. }\end{array}$ & Expt. & $\begin{array}{l}\text { Duration } \\
\text { min. }\end{array}$ & $\begin{array}{c}\text { Experi- } \\
\text { mental } \\
\text { values } \\
{ }^{\circ} \mathrm{C} . \times 10^{5}\end{array}$ & $\begin{array}{c}\text { Experi- } \\
\text { mental } \\
\text { weighted } \\
\text { mean } \\
{ }^{\circ} \mathrm{C} . \times 10^{5}\end{array}$ & $\begin{array}{l}\text { Extreme } \\
\text { varia- } \\
\text { tion from } \\
\text { the mean } \\
{ }^{\circ} \mathrm{C} . \times 10^{5}\end{array}$ & $\begin{array}{c}\text { Values } \\
\text { determined } \\
\text { graphically } \\
{ }^{\circ} \mathrm{C} . \times 10^{5}\end{array}$ \\
\hline All & values are & & & & All values are & & \\
\hline 10 & 0.05 & 3 & 70 & +11 & 12 & & $\left\{\begin{array}{l} \pm 12 \\
-13\end{array}\right.$ \\
\hline & 0.10 & 4 & 70 & +26 & 26 & \pm 0 & +26 \\
\hline & & 9 & 60 & -26 & & & \\
\hline & 0.20 & 6 & 50 & +59 & 55 & +4 & $\left\{\begin{array}{l}+55 \\
-56\end{array}\right.$ \\
\hline & & ro & 40 & -55 & & & \\
\hline & & 104 & 40 & +59 & & & \\
\hline & 0.40 & 8 & 40 & +116 & 117 & \pm 1 & $\{ \pm 117$ \\
\hline & & $I I$ & 40 & -118 & & & \\
\hline & 0.60 & $1 \sigma_{5}$ & 50 & +189 & 184 & +5 & +184 \\
\hline & 1.00 & 163 & 50 & -281 & 281 & \pm 1 & $\left\{\begin{array}{l} \pm 281 \\
-281\end{array}\right.$ \\
\hline & & 181 & 30 & -280 & & & \\
\hline & & 182 & 30 & +282 & & & \\
\hline
\end{tabular}

${ }^{28}$ Leakage coefficients like those of Table IV may be used with convenience to check the accuracy of measurements affected by accidental fluctuations of environmental temperatures. They might, obviously, be used as correction factors, so that the calorimeter could be operated in an uncontrolled outer environment; but this procedure would be less convenient than the use of the controlled environment, and less safe. Even in the case of slow measurements which involve a large change of temperature, the occasional readjustment of the closet thermostat regulator (to keep the environmental temperature always within the permissible range for negligible leakage) will be easier than maintenance of an isothermal environment, and correction for leakage into it.

The smallness of the heat loss which resulted from a very vigorous pumping by the reciprocating stirrers, disposes of the last possible objection to the use of this type of mechanism. It has already been shown that variations in the rate of development of stirring heat caused by mechanical irregularities, which have been assumed to be characteristic of these stirrers, are easily prevented (p. 909). Inasmuch as the reciprocating stirrer possesses many unique advantages in practice (Ref. 1, pp. 1915-1916) its effective insulation, thus demonstrated, is a matter of no small concern. 


\begin{tabular}{|c|c|c|c|c|c|c|c|}
\hline \multirow[t]{10}{*}{20} & \multirow[t]{2}{*}{0.10} & $15 I$ & 42 & +18 & 15 & \pm 3 & $\left\{\begin{array}{l}+14 \\
-15\end{array}\right.$ \\
\hline & & 152 & 40 & -12 & & & \\
\hline & \multirow[t]{2}{*}{0.20} & 155 & 30 & +31 & 32 & +1 & $\left\{\begin{array}{l}+32 \\
-32\end{array}\right.$ \\
\hline & & $\pi 53$ & 40 & -32 & & & \\
\hline & \multirow[t]{2}{*}{0.60} & I56 & 30 & +110 & 112 & +2 & $\left\{\begin{array}{l}+111 \\
-113\end{array}\right.$ \\
\hline & & 154 & 40 & -113 & & & \\
\hline & 0.90 & 178 & 43 & $+169^{\circ}$ & 175 & -6 & $\left\{\begin{array}{l}+176 \\
-174\end{array}\right.$ \\
\hline & 1.00 & $I \sigma_{I}$ & 40 & $+186^{c}$ & 195 & -9 & $\left\{\begin{array}{l}+197 \\
-193\end{array}\right.$ \\
\hline & \multirow[t]{2}{*}{1.10} & 170 & 31 & $+225^{c}$ & 216 & +9 & $\left\{\begin{array}{l}+219 \\
-213\end{array}\right.$ \\
\hline & & 180 & 40 & -213 & & & \\
\hline \multirow[t]{24}{*}{40} & \multirow[t]{5}{*}{0.10} & 14 & 58 & $(+4)^{b}$ & \multirow[t]{5}{*}{11} & \multirow[t]{5}{*}{ \pm 1} & \multirow[t]{5}{*}{$\begin{array}{l}+10 \\
-12\end{array}$} \\
\hline & & Ig & 50 & +12 & & & \\
\hline & & 24 & 40 & -10 & & & \\
\hline & & 64 & 50 & +10 & & & \\
\hline & & 102 & 40 & +10 & & & \\
\hline & 0.15 & 18 & 50 & +16 & 16 & & $\left\{\begin{array}{l}+16 \\
-19\end{array}\right.$ \\
\hline & \multirow[t]{4}{*}{0.20} & 15 & 40 & +30 & \multirow[t]{4}{*}{25} & \multirow[t]{4}{*}{+5} & $\{+24$ \\
\hline & & 20 & 40 & +30 & & & \\
\hline & & 25 & 60 & -25 & & & \\
\hline & & 65 & 40 & +27 & & & \\
\hline & 0.30 & 30 & 40 & +46 & 40 & +6 & $\left\{\begin{array}{l}+39 \\
-41\end{array}\right.$ \\
\hline & \multirow[t]{6}{*}{0.40} & 16 & 50 & +56 & \multirow{6}{*}{57} & \multirow{6}{*}{+5} & +56 \\
\hline & & 22 & 40 & $(+50)^{\circ}$ & & & \\
\hline & & 26 & 50 & -60 & & & \\
\hline & & 67 & 43 & +61 & & & \\
\hline & & 103 & 40 & +58 & & & \\
\hline & & 139 & 60 & +62 & & & \\
\hline & 0.60 & $2 I$ & 40 & +92 & 97 & -5 & $\left\{\begin{array}{l} \pm 96 \\
\pm-87\end{array}\right.$ \\
\hline & \multirow[t]{3}{*}{0.80} & 28 & 70 & +143 & \multirow[t]{3}{*}{142} & \multirow[t]{3}{*}{+4} & +143 \\
\hline & & 33 & 40 & -142 & & & \\
\hline & & 140 & 60 & +146 & & & \\
\hline & \multirow[t]{2}{*}{1.00} & 27 & 40 & +197 & \multirow[t]{2}{*}{194} & \multirow[t]{2}{*}{+3} & $\left\{\begin{array}{l}+194 \\
\pm 194\end{array}\right.$ \\
\hline & & 34 & 50 & -194 & & & \\
\hline & 1.20 & 20 & 38 & +244 & 250 & -5 & +250 \\
\hline
\end{tabular}

"A comparison of the experimental values and those graphically determined will indicate the degree to which the curves were "smoothed out" in plotting. The weighted mean was found by a critical examination of all immediate data; and excepting where single points were but once determined, without reference to the curve. It will be seen that the values graphically determined agree very well with the weighted mean values. Each extreme variation from this mean therefore, is greatly in excess of the probable error.

${ }^{b}$ This value shows a result of capillary friction.

c These values were all affected by large calibration corrections which changed the values to which they were applied by amounts about twice as great as the usual divergences between them here recorded. Of these, Nos. 161,178 and 179 were also affected by time reading errors.

The bracketed values were not considered in reading variations from the mean.

Expts. 64,65 and 67 were made with an initially saturated air gap, which contained, however, no free water. The measurements were made with various assemblages in which the calorimeter surfaces were at times highly reflecting, at others perceptibly dull, between January 8 and May 10, 1921. 
mm. wide; and with each of these, the thermal leakage under several constant heads between $\pm 0.05^{\circ}$ and $\pm 1.20^{\circ}$ (cf. p. 914) was determined for intervals of from 30 to 120 minutes. ${ }^{29}$

The complete data are given in Table $\mathrm{V}$, and the corresponding leakage curves in Fig. 3. It is apparent that convection here causes a surprisingly small divergence from Newton's law, even with the widest gap under its greatest temperature gradient, and that this is the same for leakage in both directions. It follows at once that with the closed calorimeter all these gaps, even the widest, may be safely used in any adiabatic determination ${ }^{30}$ conducted according to the procedure already adopted, even under rough control. ${ }^{31}$ But another consequence is of much greater

${ }^{29}$ The most precise determinations were made in groups of 4 to 8 , always preceded and followed and occasionally interrupted by hour-long tests of the behavior of the system under isothermal conditions, and were thus proved to be unaffected by any influences other than the thermal heads. The results so obtained were checked by separate measurements scattered throughout the whole period of experimentation; which showed not only that the original procedure involved no accidental errors of any consequence, but that the surface changes incident to long-continued manipulation were insufficient to affect the constancy of radiation leakage for a given head.

In the first determinations, the calorimeter, for lack of adequate mechanisms for stirring speed control, was stirred intermittently; and correction was subsequently made for the small errors of lag thus introduced, by later and very precise determinations in which, under conditions otherwise identical, stirring was both intermittent and continuous (p. 914). The results of these later measurements are given in Table III, but not in Table $V$, since because they were carried out with a reconstructed and replated calorimeter, they were not strictly comparable with the preceding. Considered as a separate set of values, their relations are in complete accord with those of Table $\mathrm{V}$.

In a few measurements the calorimeter was imperfectly sealed and showed evaporation leakages in the equilibrium tests, which were nearly constant for any group of determinations and were thus safely corrected for. In all measurements temporary irregularities were detected, and all errors of transmission lag were quite eliminated by determining the partial leakage rates at 10 -minute intervals and by selecting for record only those ranges over which the flow was constant within thermometric error. The careful selection of well determined end temperatures (Ref. 4), the checking of suspected results by calculation of leakage over overlapping segments of the total range, and the fixing of initial and final temperatures by two or more readings at 1 -minute intervals, often reduced thermometric error closely to the least personal error of observation; while the duration of the measurements made its infiuence upon the precision as small as was desirable or practical. The calibrated temperature readings were invariably corrected in ten-thousandths of a degree for stirring heat, for excess leakage between calorimeter and bath, for leakage to and from the outer environment, and for exposure of the thermometer threads. The sum of these corrections rarely exceeded $0.001^{\circ}$, and thus included no significant errors. Time-reading errors were negligible excepting in measurements of the greatest leakages.

The aggregate temperature range was $19.3^{\circ}$ to $20.5^{\circ}$. Within this range, as duplicate determinations showed, change of mean temperature had no measurable effect.

Throughout the whole series there was no evidence of exceptional behavior.

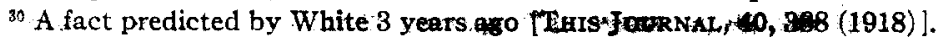

3: Ref. 1, p. $1920 f$. The present curves show that with the widergaps thelloakage 
practical importance. The leakage through the widest $(40 \mathrm{~mm}$.) gap is so very small that with it, if the outer environmental and bath temperatures be made to fluctuate with fair regularity, respectively within

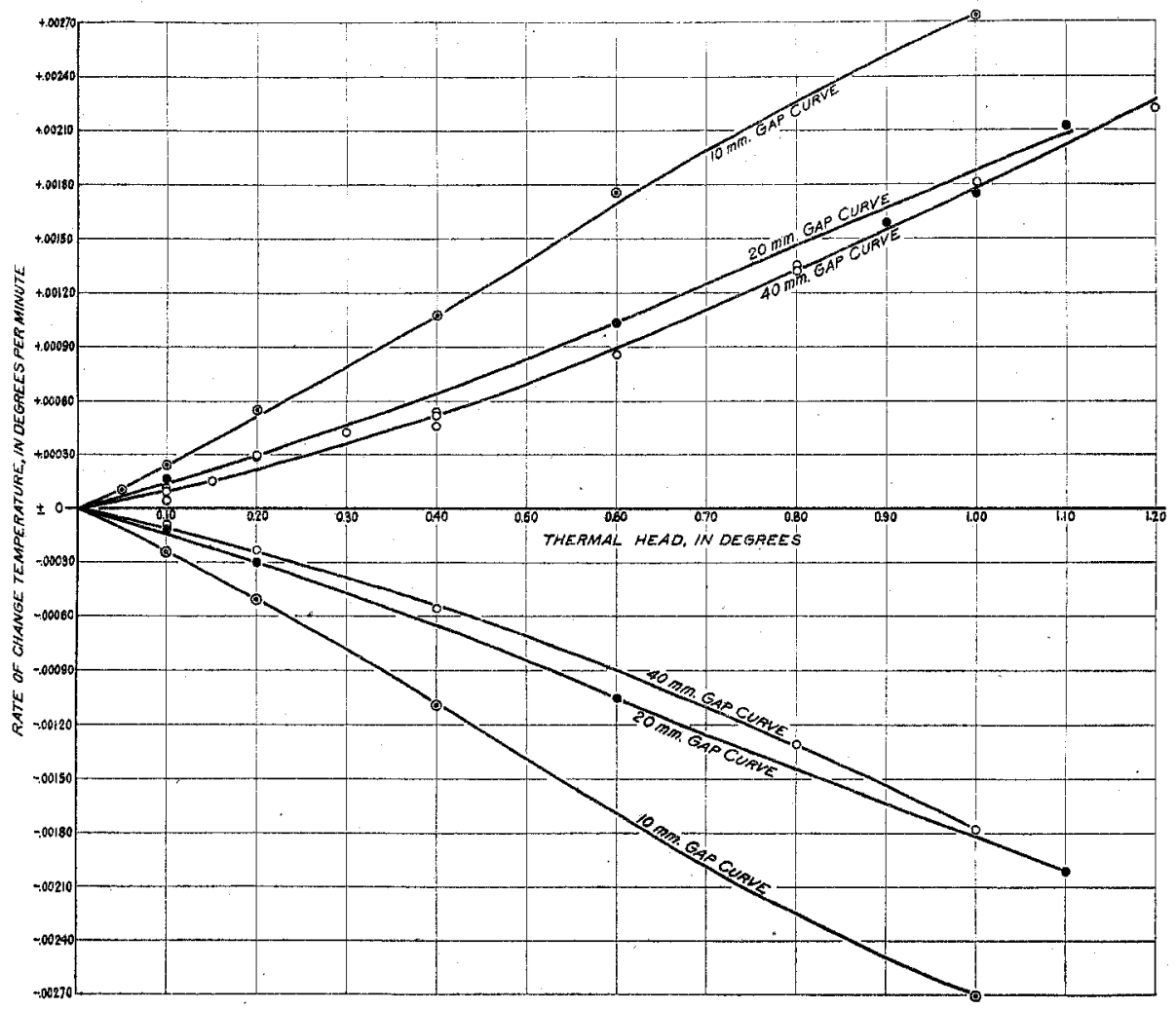

Fig. 3.-Thermal leakage between closed calorimeter and bath: relation to thermal head. $\pm 0.02^{\circ}$ and $\pm 0.01^{\circ}$ of the calorimetric temperature, it is possible to maintain for hours a condition which with respect to final leakage effect is equivalent to strict adiabaticity. ${ }^{32}$ It is not necessary, however, to mainis measurably proportional to thermal head for bath fluctuations as great as $=0.10^{\circ}$. The influence of the heat capacity of the air gaps and of all transmission lags is negligible (see Refs. 16, 17).

${ }^{32}$ Under a head of $0.2^{\circ}$ continuously maintained, leakage to or from the outer environment will approximate, with assemblages similar to that here used, 0.6 gramcalorie in 10 hours (Table IV and Ref. 11), and under a constant bath head of $0.01^{\circ}$, leakage through the jackets will be, in the same time, $8.2 \mathrm{gram}$-calories (Table V, etc.). In the present experiments the corrections for this aggregate leakage effect during 1 hour or more were repeatedly reduced to 0.2 gram-calorie and often to less than $0.1 \mathrm{~g}$.-cal. by maintaining fluctuating external temperatures. With calorimetric systems of 1000 g.-cal. units capacity, this leakage corresponds to a temperature change of not more than $0.0002^{\circ}$. There is no reason why it should be much greater during longer periods of time. 
tain such careful control; since, even if only ordinary precautions be observed, the leakage will surely be less than one calorie, and the errors of its estimation quite negligible. Of nearly equal interest is the relation shown by these curves between total leakage and gap width. This is brought out more clearly by the graph of Fig. 4. The effectiveness of the air insulation increases less rapidly for a given increase in width as the gap becomes wider, in such wise that optimum insulation for small thermal heads is practically reached at the $40 \mathrm{~mm}$. gap width. It is

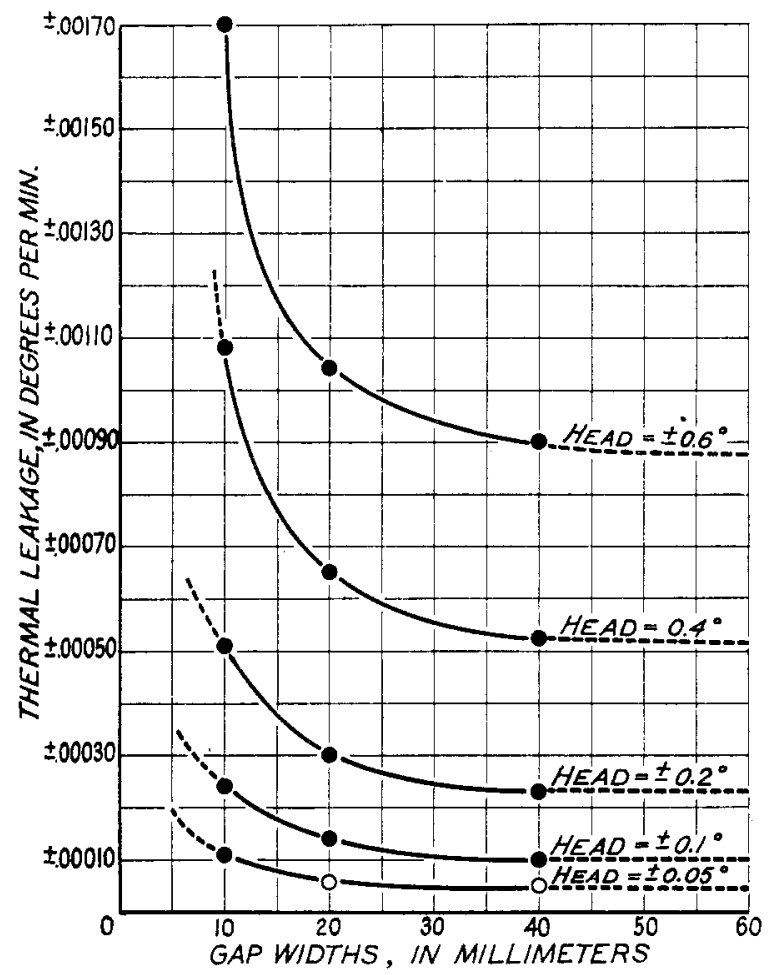

Fig. 4. -Thermal leakage between closed calorimeter and bath-relation to gap width.

clear, therefore, that in adiabatic calorimetry there will be no significant advantage in using gaps of greater widths than this, excepting with very large calorimeters.

An analysis of the several thermal effects involved in this leakage yields interesting results. Of the three factors of thermal leakage, conduction and radiation vary directly as the thermal head (for the small ranges here involved), while convection varies more rapidly. If, therefore, (in Fig. 3) straight lines be drawn tangent to the curves of total leakage at their origins, the ordinate distances between these lines and 
the corresponding curves measure the convection leakage. In the present instance, the convection leakages were thus determined, the tangents being drawn through the origins and the determined points at $0.05^{\circ}$ head on each curve. ${ }^{33}$ It was thus discovered that, while convection over the whole range measured increased more rapidly than the thermal head, the rate of increase in all cases diminished with increase in head. The initial rate of increase was smaller and the rate of its diminution was greater, the smaller the gap width. This general behavior, hardly perceptible to the eye in the curve for the $40 \mathrm{~mm}$ : gap and in the first half segments of the other curves, is clearly apparent in the curve for the $10 \mathrm{~mm}$. gap, which shows distinct reverse flexure at about $0.6^{\circ}$ head and becomes parallel to its tangent a little below $1.0^{\circ}$ head. The reversal of the $20 \mathrm{~mm}$. gap curve occurs at a head somewhat beyond $0.6^{\circ}$ and is less marked. It may be presumed that the $40 \mathrm{~mm}$. gap curve would show similar flexure beyond $1.2^{\circ}$ head. Thus, with increase of thermal head, the curves of the several gaps diverge less and less. An interesting consequence of this behavior is that at about $1.0^{\circ}$ head, the total leakage through the $20 \mathrm{~mm}$. and $40 \mathrm{~mm}$. gaps is the same (Fig. 3). The quantitative relations are shown in Tables VI and VII. ${ }^{34}$ From Table VI it is apparent that with the $40 \mathrm{~mm}$. gap, convection varies over nearly the whole range as the square of the thermal head, exactly within measurement error; but that with the 10 - and $20 \mathrm{~mm}$. gaps, it varies respectively as the mean 1.4 and 1.8 powers of the head. The relations of convection leakage to gap width are better shown in Table VII. It is there made evident that at small heads the convection tends to decrease, while at greater heads it increases with increase in gap width. Broadly speaking, at $0.2^{\circ}$ head the convection varies inversely as the square root, and at $1.0^{\circ}$ head directly as the $2 / 3$ power of the gap width; and the data indicate that at greater heads the convection varies with an accelerated rate of change as higher powers of the gap width. With reference to previously determined leakages under greater thermal heads than

33 'The curves were' all measurably linear up to this head. When tangents were constructed through points $0.0001^{\circ}$ lower on the $0.05^{\circ}$ ordinate, they stood away from the curve and were perceptibly too low; and when they were drawn with half this divergence (the probable error of construction), the essential relations hereafter summarized were not significantly affected. (Tables VI, VII and VIII.).

${ }^{34}$ In the calculations, the convection leakages were all corrected for variation of the actual air gap widths from exact dimension, on the basis of the relations exhibited by the actual data (Table $\mathrm{V}$ ) and in accordance with the assumption that convection per unit effective area was twice as great in the basal as in the lateral gaps. Cf. White, Ref. 35, p. 755. This correction involved necessary assumptions, especially with respect to the magnitudes of the convections from the vertical surfaces; but when these were such as to malke the corrections maximal, the original values were not affected beyond the range of possible experimental error. The corrections of convection leakage for dimension were thus considered negligible. 

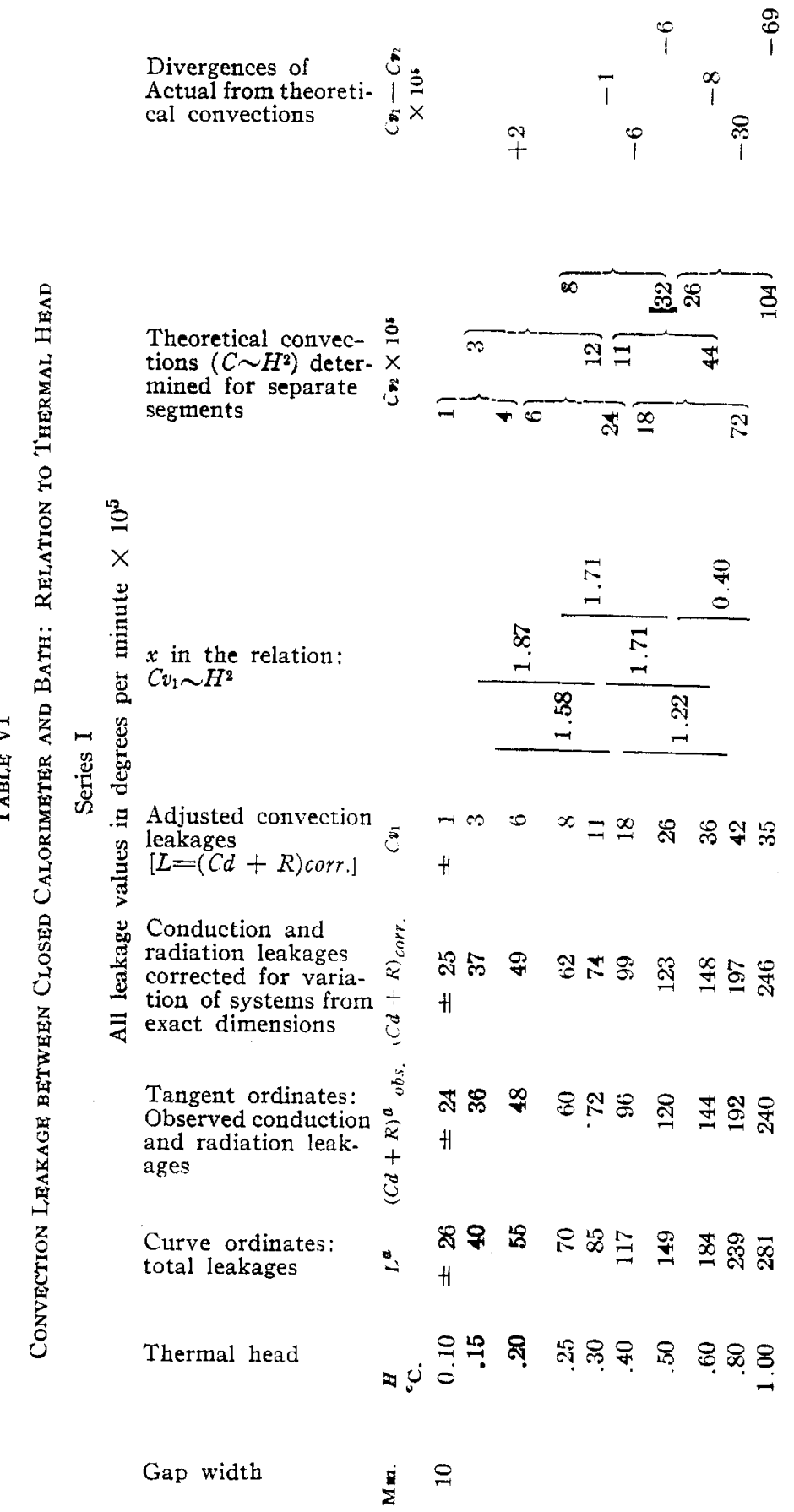

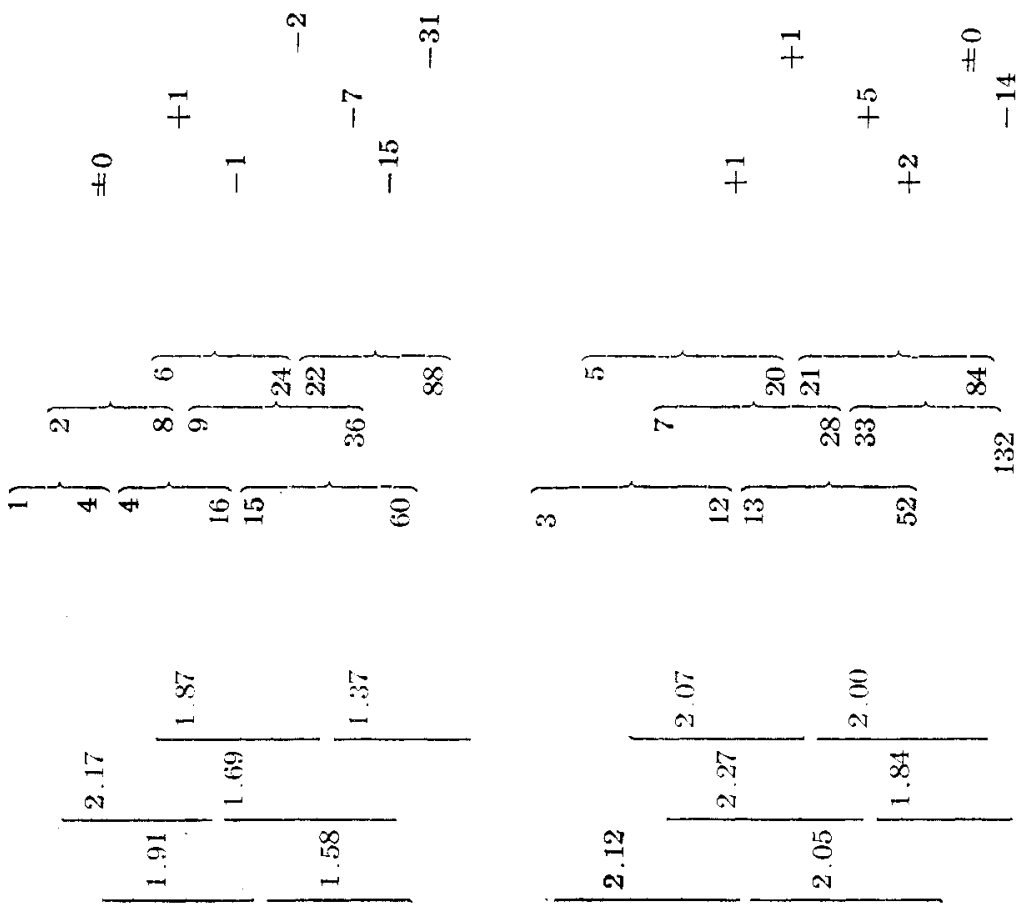

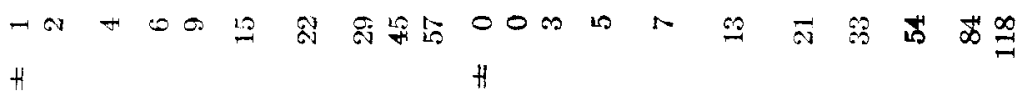
ป一⿻ H

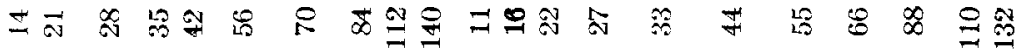
Hit

12 ลำ H H

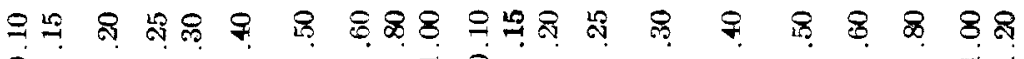
$\circ$ శึ 
a The total leakage values are affected by corrections for intermittent stirring lags ( $c f$. Table III). If these be not applied, the relative values of $x$ above will be the same as those determined on the assumption that the lag is proportional to thermal head, and will have the following successive values: For the 10mm. gap: 1.87, 1.50, 1.64, 1.63, $1.37,0.82$; for the $20 \mathrm{~mm}$. gap: $2.00,1.81,1.74,1.76,1.62,1.37$; for the $40 \mathrm{~mm}$. gap: $2.12,2.00,2.10,1.97,1.96,1.84$. The inferences drawn from the data will, therefore, not be affected by doubt in consequence of possible errors in the estimation of lag.

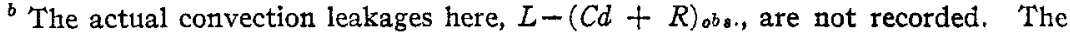
conduction and radiation leakages determined by the tangent constructions, $(C d+R)_{a b}$., were corrected for variation from exact dimension (so as to represent this leakage for gaps exactly 10,20 , and $40 \mathrm{~mm}$. wide) on the supposition that conduction was uniform and normal to the calorimetric surfaces. This neglect of "end effects" introduced no significant error into the small corrections; and since the dimensional error had no measurable effect on the convection leakage, the adjusted values of the convection were determined with sufficient precision as $L-(C d+R)_{\text {corr. }}$. The final relations determined apply, therefore, within measurement error to gaps of exact 10,20 and $40 \mathrm{~mm}$. dimension.

\section{TABLE VII}

\section{CONVECTION LEAKage BETWEEN Closed CALORIMETER AND Bath RELATION TO GAP WIDTH}

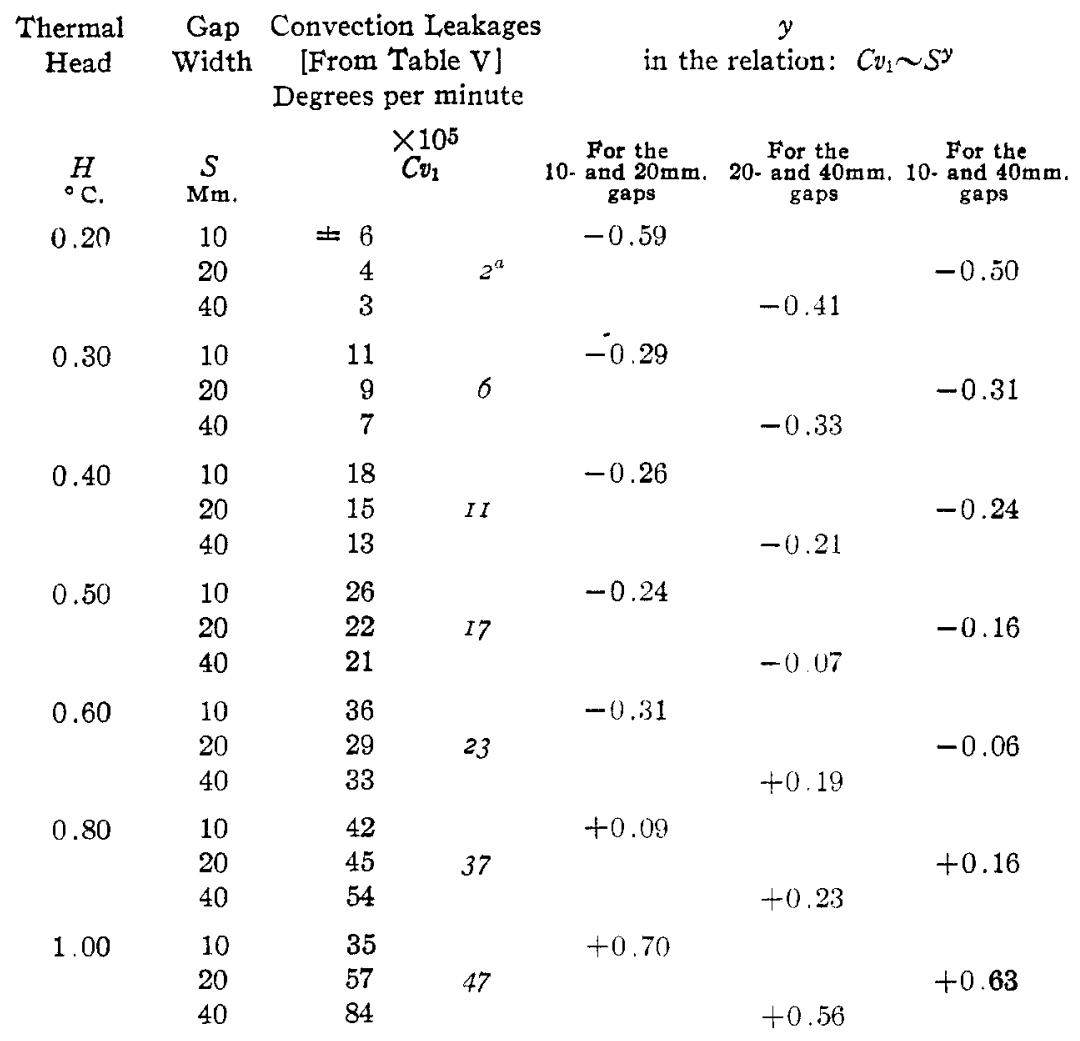

a Figures in italics refer to the adjusted tangent ordinates of the $20 \mathrm{~mm}$. gap (Ref, 42). 
these, ${ }^{35}$ this whole behavior seems sufficiently strange to appear questionable in the absence of complete assurance as to the sufficient precision of the data. When this precision is not too favorably estimated, it appears that the falling off in the acceleration of the convection with increase of head is undoubtedly real; and that the initial diminution of convection with increase of gap width, while not rigorously proved, appears to be probable-or, more definitely, that the absence of any measurable increase of convection with gap width at the smaller heads is certain. ${ }^{36}$ The phenomena as a whole suggest that two effects are operative here in retarding the convection, which, if normal, would increase continuously both with thermal head and with gap width. One of these increases with thermal head, and at any given head, is greater in the narrower gap; the other has its greatest relative effect when the head is very small, and increases with gap width. The present data are insufficient to support any definite statement as to their probable causes. ${ }^{37}$ It suffices to remark the fortu-

${ }_{35}$ White, Phys. Rev., 10, 750-751 (1917).

${ }_{36}$ The probable error of each determined leakage is not greater than $0.001^{\circ}$ in 50 minutes, or $0.00002^{\circ}$ per minute. This value, which is twice the thermometric reading error, and approximates that of temperature range determination, might in this case be assumed a priori. It is, however, vouched for by the concordance of duplicate measurements for almost every leakage. These show an extreme variability from the mean of from $0.00001^{\circ}$ to $0.00006^{\circ}$, which averages $0.000035^{\circ}$ per minute for all heads less than $1^{\circ}$ (Table V); and their separately weighted averages are confirmed within $0.00001^{\circ}$ per minute by the independently constructed curves, which in their smoothing out merely define those possible values for each head which represent a consistent continuous variability of leakage with increase of head.

This measurement error only could make doubtful the variability of leakage with increase of head (Table VI), since error in the determination of the curve tangent is proportional to the head, and the stirring lag correction is certainly proportionally no greater at high heads than at low. If it be applied to the convection leakages of the two narrower gaps so as to produce in each case its maximum possible effect in compensating the observed diminution of convection rate with increase of head (i.e., if in each case it be added to the greater and substracted from the less of the convection rates at high and low heads) this diminution persists in the $10 \mathrm{~mm}$. gap leakage, and is on the average barely compensated in the $20 \mathrm{~mm}$. gap leakage.

An analagous application of the maximal measurement error to the determined variability of convection leakage with increase of gap width (Table VII) results in an exact compensation of the observed diminution at $0.4^{\circ}$ head. At greater heads, obviously, the effect is less: at smaller heads it is indeterminate, since the leakages themselves are of similar magnitude and are highly uncertain; although the dependability of their approximate relations is vouched for by the general consistency of the complete data. This variability, therefore, is also unaffected by error of observation. The possibility of its invalidation by error in the tangent constructions (which define independently for each gap the mean convection leakage) is shown further on to be negligible (Ref. 42).

It thus appears almost certain that both variabilities are real.

${ }^{37}$ The phenomena appear less extraordinary, however, if possible causes be imagined. The first effect might result from a retarding turbulence caused by the con- 
nate circumstance that both effects are calorimetrically advantageous, since they obviously occasion the small divergence of the total leakage relations from Newton's law already noted.

Simultaneously with the convection leakage, that of radiation and conduction together had been determined. To separate these two leakages it was necessary to estimate that of conduction. Since there is no convenient mathematical procedure for such determination in the case of completely enclosed systems, the conduction leakage was found experimentally. Advantage was taken of the fact that the distribution of temperature throughout a space bounded by two closed isothermal surfaces is similar to the distribution of potential within the same space bounded by equipotential surfaces. ${ }^{38}$ It follows from this that for any particular system, the heat flow for the one condition is proportional to the electric force for the other, at any point; and that the total heat flow between the isothermal surfaces is proportional to the electrical capacity of the same system used as a condenser. For the calorimetric system, therefore, the heat conduction per degree temperature head is $4 \pi C k / K$, where $C$ is the electrical capacity in centimeters, $K$ the dielectric constant $(=1$ for air, as in the present case) and $k$ the thermal conductivity (in the present case $=0.000058$ for air at $\left.20^{\circ}\right) ;{ }^{39}$ the factor $4 \pi$ being necessary to reduce the electrical capacity to equivalent units. ${ }^{40}$

The electrical capacity of the present $40 \mathrm{~mm}$. gap system (that in which the "end effects" were greatest) was determined in the radio laboratory of Columbia University by the method of substitution in a resonant circuit loosely coupled with a high frequency generator. It was found to be 24 micro-micro farads or $21.5 \mathrm{~cm},{ }^{41}$ and yielded the value 0.0156 gram-

figuration of the gap, which is more than slightly modified by the presence of the necessary chimneys and supporting frame of the calorineter. Such turbulence would be likely to be greater at greater heads and in the narrower gaps. The second effect might be caused by irregular distribution of heat on the jacket wall. This would occasion slight convection currents roughly parallel to the surfaces, which would tend to cause a general drifting of air across the normal stream lines. Its effect would be noticeable only in gaps of sufficient width, but would be compensated by the increase of normal convection with increase of gap width; and since it would be independent of the head, it would be noticeable only under small heads. A like effect might perhaps be produced by protracted transmission lags, which would be greater at smaller heads and in wider gaps, but which would be similarly compensated.

${ }^{38}$ This of course is a special instance of a more general analogy.

${ }^{39} \mathrm{E}$. Mueller, Wied. Ann., 60, 82 (1897).

10 This relation is very clearly seen in the simple case of a system of two parallel areas on infinite plane surfaces. Here, heat conduction per degree temperature head $=(A / d) k$, and electrical capacity $=(A / d) K \div 4 \pi$, where $A$ is the area of each surface and $d$ the distance between the two (in centimeters); whence the heat conduction per degree temperature head $=4 \pi C\left({ }^{k} / K\right)$ (calories per second, when $C$ is the capacity in centimeters).

¿1 The separate measurements made with different dispositions of the intermal leads 
calorie per second, corresponding to $0.00076^{\circ}$ per minute for the conduction leakage, and 0.0070 gram-calorie per second, or $0.00034^{\circ}$ per minute for the radiation leakage. This radiation leakage, assumed to be the same in every measurement made under $1^{\circ}$ head and to vary like the conduction proportionally with the head, then yielded the complete series of conduction leakages; and in combination with the other data of measurement, the ratios of convection, conduction and radiation leakage under all of the established conditions. The complete data are given in Table VIII. ${ }^{42}$ The leakage ratios there given bring out very clearly the calorimetric advantage of the wide $(40 \mathrm{~mm}$.) gap. With this gap the conduction leak-

yielded the values $25.0,24.0,23.3,24.4 \mathrm{~m} . \mathrm{m}$.f. The accuracy of measurement seems to he considerably better than $5 \%$ and might, without doubt, be improved, were a closer approximation necessary.

It is clear that the above procedure furnishes a convenient method for determining the thermal conductivity of any closed insulating space, whatever its configurat:on.

Since the calorimeter here used was of typical form (a cylinder of which the height is about three times the radius) and since the air gaps surrounding it were nearly symmetrical, the data obtained may serve to define an empirical rule for the approximate estimation of conductance in similar systems. The conductance of the $40 \mathrm{~mm}$. gap system was 0.93 times, and that of the $10 \mathrm{~mm}$. gap system was 0.87 times the conductance of two plane areas on infinite plates the gap width apart, each equal to the total surface of a cylinder having the mean radius and height of the gap surfaces. With close approximation then, the conductance of a closed cylindrical system of the most convenient form (in which the height of the calorimeter is about three times, and the uniform gap width no greater than $3 / 4$ of the calorimeter radius) may be very quickly computed with reference to this cylinder of mean dimensions. For all such systems, the conductance under $1^{\circ}$ head $=0.9 \frac{2 \pi R(R+H)}{d} k$ calories per second, where $R$ and $H$ are the mean radius and height of calorimeter and jacket and $d$ the gap width in centimeters, and where $k$ is the thermal conductivity of the insulating material.

The writer desires in this place to acknowledge his great obligation to Professors $\mathrm{H}$. W. Webb and J. H. Morecroft for their kindness in arranging for and in carrying out the electrical measurements.

42 A valuable criterion for the correction of previous estimates of the combined radiation and conduction leakages was provided by the calculation of minimal values for the conduction leakages. On the assumption that all conduction was normal to the surfaces, and that it varied, therefore, inversely as the gap width between basal surfaces and inversely as the logarithm of the ratio of gap radii between the lateral surfaces, the minimal conduction leakages for the several systems used were calculated from the actual gap dimensions. They were found to be for the $10 \mathrm{~mm}$. gap, $0.00199^{\circ}$, for the $20 \mathrm{~mm}$. gap $0.00112^{\circ}$ and for the $40 \mathrm{~mm}$. gap, $0.00062^{\circ}$ per minute. These minimal values were consistent with the experimentally determined leakages of the $10 \mathrm{~mm}$. and $40 \mathrm{~mm}$. gaps, but not with those of the $20 \mathrm{~mm}$. gap (Table VIII). It thus appeared that the tangent of the $20 \mathrm{~mm}$. gap curve had been drawn too low. The values of the convection and conduction leakages determined by it were, therefore, corrected, within possible error of previous estimation (Ref. 33) by increasing its intercept at $1^{\circ}$ head from $0.00140^{\circ}$ to $0.00150^{\circ}$ per minute; and this having been done, the derived values of the conduction leakages became consistent. These and all corresponding adjusted values are printed 


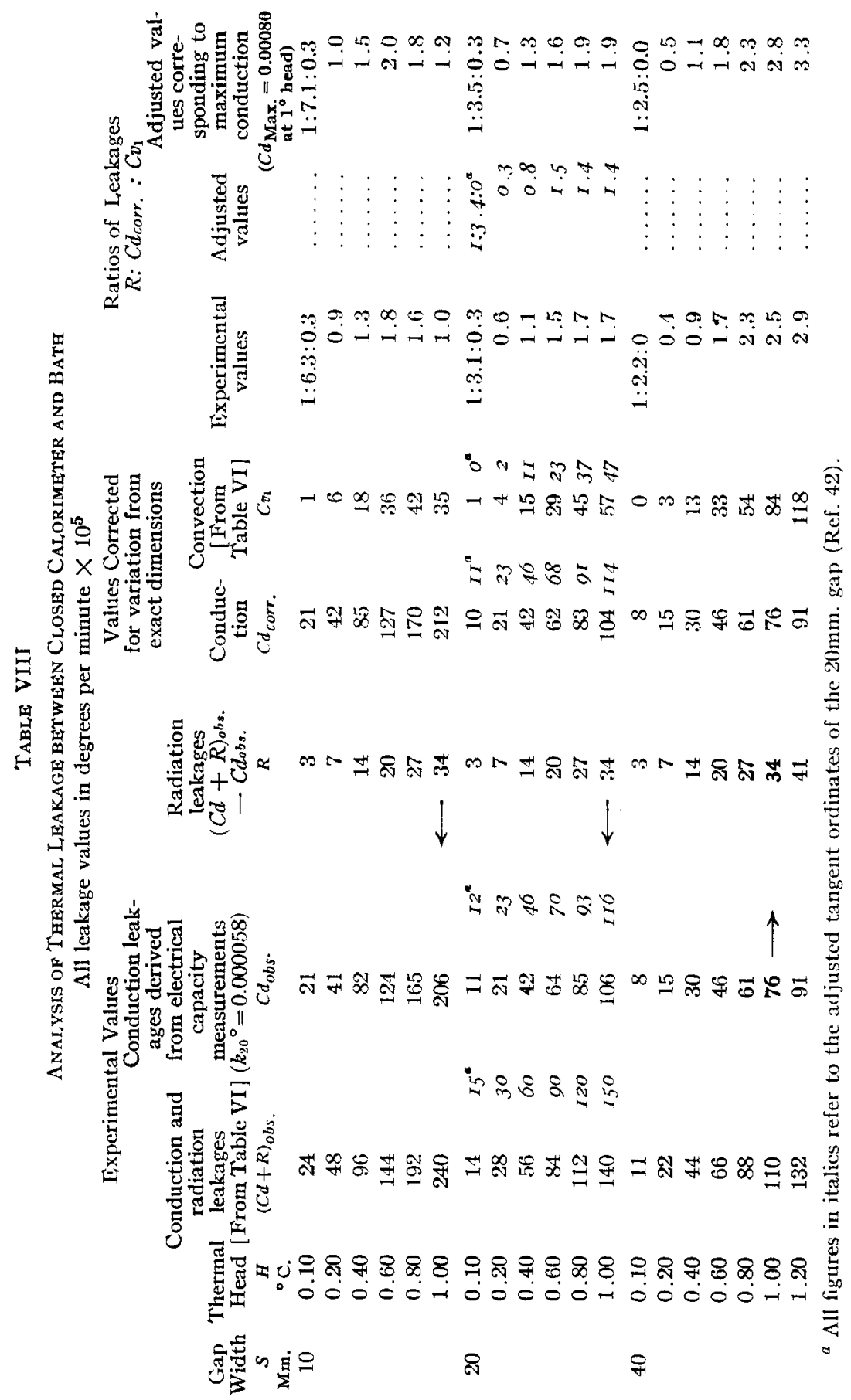


age at all heads is reduced nearly to twice that of the inevitable radiation; while the convection leakage, which remains less than that of corresponding conductions at all heads below $0.80^{\circ}$, becomes negligible below $0.10^{\circ}$ head. It follows that in adiabatic practice this air gap provides an insulation almost a third as effective as a perfect vacuum gap, and thus renders more or less illusory any imagined advantage to be gained in adiabatic calorimetry by the use of practicable vacuum insulations.

\section{Effects of Condensation of Moisture on Calorimeter Surfaces}

The regularity in the behavior of the closed calorimeter with respect to thermal leakage, above described, was conditioned by the maintenance of a practically dry gap. The presence of even a small quantity of liquid water in the gap always caused upon closure a rapid saturation, followed by a long-continued rise in the temperature of the calorimeter, which apparently was due to the condensation of water upon it. This effect, the extreme duration of which was unanticipated, was obviously a possible source of very serious error in measurement, and was examined with some care.

The calorimeter jacket cover, which like that of the calorimeter carried extra apertures designed for use in other measurements, was fitted with a glass pipet through which water could be quickly withdrawn from the calorimeter at any time, and with bent tubes through which it could be delivered into the gap without wetting the calorimeter surface, both arrangements being so contrived that excepting during the half minute of manipulation, the calorimeter and gap would remain effectively insulated. The isothermal condition having been maintained for an hour or more, water in quantity insufficient to affect measurably the heat capacity

in italics in Tables VII and VIII. It will be seen that they lead to the same inferences as the original data, in all cases (cf. Ref. 36).

By a comparison of the calculated minimal values given above with the corresponding experimental values of Table VIII, it will be evident that the "end effects" occasion about $18 \%$ of the whole conduction leakage in the $40 \mathrm{~mm}$. gap system, but are negligible with the other systems. It follows that for systems of similar configuration with air gaps no wider than $20 \mathrm{~mm}$., the conduction leakage may be calculated with a precision s:Afficient for usual purposes directly from the gap dimensions, on the assumption that all lines of heat flow are normal to the gap surfaces.

This conclusion was substantiated, and several errors of previous approximation were checked, by a derivation of the coefficient of conductivity for air at $20^{\circ}$ from the adjusted values of the combined radiation and conduction leakages for the 10- and $20 \mathrm{~mm}$. gaps, and from the ratio of the corresponding minimal conduction leakages calculated from that of the gap dimensions.

Experimentally, in degrees per minute: for the 10 - and $20 \mathrm{~mm}$. gaps at $1^{\circ}$ head, $\left(C d_{10}+R\right)$ corr. $=0.00246$ and $\left(C d_{20}+R\right)$ corr. $=0.00148$ (Table VIII); whence $C d_{10}-C d_{20}$ $=0.00098$. By calculation from corresponding ideal dimensions $C d_{10} / C d_{20}=0.53$. Hence $C d_{10}=0.00208$ and $C d_{20}=0.00111$ degrees per minute; and $k_{20}^{\circ}=0.000057$, in both cases. 
of the system was withdrawn and quickly transferred to the gap. An immediate rise in temperature was observed, which continued at a practically undiminished rate for an hour, and which, after several hours, was still increasing; it was, moreover, the same whether the bath temperature fluctuated about that of the calorimeter or was held continuously below it. It is clear that this temperature rise could have been due to nothing but superficial heating caused by the condensation of water on the surface of the calorimeter. In the first tests, the calorimeter surface, though fairly clean, was not brightly reflecting. It was later thoroughly cleaned, particular care being taken to remove the last traces of possible hygroscopic contamination and of grease. ${ }^{43}$ The test was then repeated: the temperature effect was the same, though diminished. After it had become imperceptible, the surfaces were exposed and allowed to dry by evaporation. Another repetition of the test then yielded the same result. In later trials the dry surface was slightly greased by a very minute quantity of vaseline, and then completely covered with a thin layer of vaseline. The effects were precisely similar, and with the well greased surface were measurably the same as with the cleanest. It was thus made probable that the effect was not due to the presence of chemically reacting or of hygroscopic material on the surface, and that it could not be prevented or significantly accelerated by attempting to reduce the magnitude of the adsorption. Precisely similar effects were observed with the open calorimeter, the surface of which was dry. In a dry gap, its temperature naturally fell as a consequence of evaporation, but when the gap was wetted as in the previous tests this thermal effect was suddenly reversed. With both systems the entire effect was eliminated by wiping the calorimeter vessel all over with a chemically clean damp cloth, allowing it to stand under a bell jar until the surface showed very few adhering drops, and enclosing it in a water-saturated gap. It is not certain that the more rapid evaporation from adhering drops was significant, though the results of one or two measurements indicated this. The possibility of irregularity from this cause would better, however, be thus avoided.

The character and magnitude of these effects are shown in Table IX. These data indicate that very serious errors are likely to occur in calorimetric measurement when dry calorimeter surfaces are placed in wet gaps, and point specifically to the dangerous disturbances which may result from gasket leakage, or from accidental spattering of calorimeter water

${ }^{43}$ The apparently clean surfaces were rubbed and washed with benzene, then with pure soap, scrubbed with clean wet cloths and thoroughly drenched with grease-free distilled water. They were afterward rubbed with chemically clean cloth saturated with alcohol, washed with alcohol, again drenched with water, and dried in dust-free air. The last adhering water film gave no indications of the presence of grease, and the finally dry surface was brightly reflecting and apparently untarnished. The cleaned calorimeter was kept under a bell jar and was invariably handled with a clean cloth. 
TABLE IX

EFFects of Condensation of Motsture on Calorimeter Surfachs

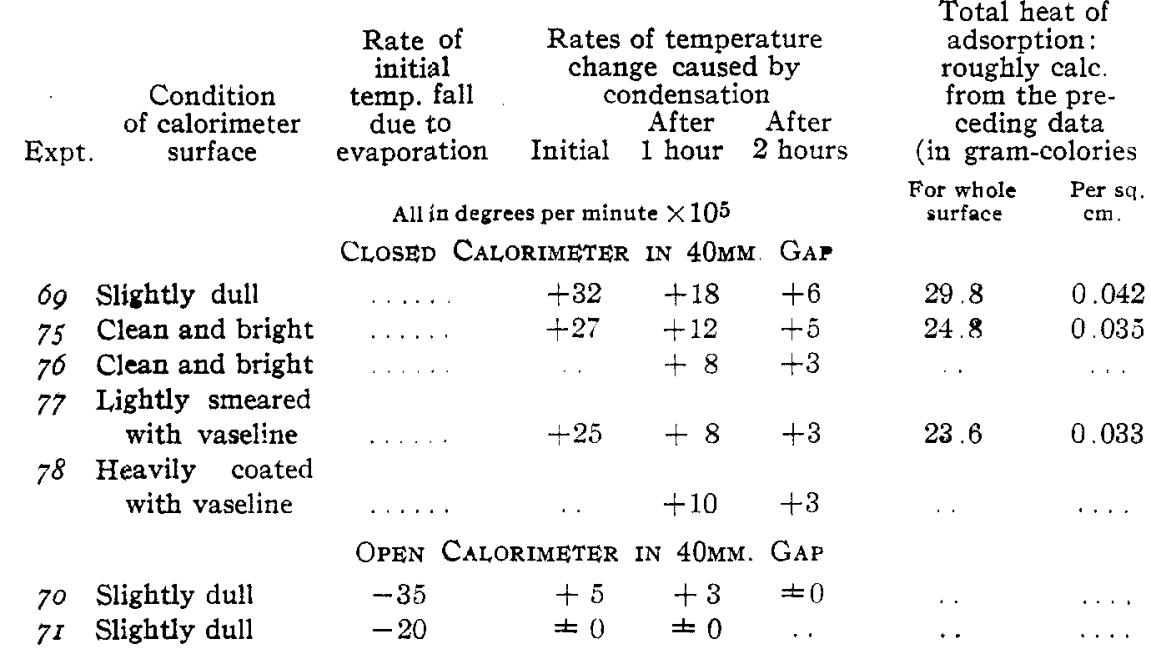

during assemblage. Inasmuch as the effect is not only greatest, but is also variable during the first 30 minutes, it is likely to introduce error into the measurement of rapid as well as that of slow changes. The effect of the evaporation of accidental water from a calorimeter surface into a dry gap, and that of the chemical absorption of the gap moisture by hygroscopic substances on the calorimeter surface have been previously commented upon. ${ }^{44}$ The present observations appear to show that any accidental water in the gap will occasion significant irregularities of behavior, whether hygroscopic substances are present or not. ${ }^{45}$ With the closed calorimeter it is best to keep all gap surfaces scrupulously dry, and in this case it becomes doubly important to make the vessel vaportight, inasmuch as, if evaporation occurs, some of the escaped moisture will be deposited on its outer surface. Since the phenomenon is one of 44 White, Phys. Rev., 31, 575 (1910).

${ }^{45}$ The data of Table IX indicate that even when the calorimeter surface is clean and bright, the total amount of water adsorbed is $0.00006 \mathrm{~g}$. or more per sq. $\mathrm{cm}$. This quantity is very much greater than that observed by White (Ref. 44) to be adsorbed by clean nickel surfaces, and is comparable to the adsorption on tarnished copper which he detected. This large discrepancy cannot be ascribed to the smaller sensitivity of the calorimetric measurements. It is not impossible that the condensations here observed were due in part to very slight contamination, though in view of the precautions taken, (see Ref. 43) this seems unlikely. On the other hand, White's adsorptions may have been incomplete. It is highly desirable that this discrepancy be explained. Meanwhile, the practical consequences of the present observations remain unaffected by any doubt. The calorimeter surfaces in these tests were certainly as clean as very careful manipulation permitted them to be. The phenomena observed, therefore, whether they were due wholly or only in part to the simple adsorption of moisture, may be accepted as characteristic causes of disturbance in any practical calorimetry. 
adsorption, such condensation will occur before the gap reaches saturation, and it will reduce the thermal effect of the evaporation in an indeterminable way. With the open calorimeter the only recourse is to wet the surfaces of the vessel in the manner described above and to enclose it in a saturated gap.

\section{Leakage between Open Calorimeter and Bath}

Measurements of leakage were carried out with the open calorimeter in all respects similar to those made with the closed calorimeter. The behavior thus exhibited was so complicated that any description of it sufficiently minute to prove the validity of the inferences drawn from its analysis would probably not be justified by their practical importance. These inferences will, therefore, be only briefly summarized in this place; the evidence upon which they rest, if it ever seems necessary or advisable to present it, will be given in full elsewhere at another time. ${ }^{46}$

With an open calorimeter in a gap initially dry, the thermal disturbances, which are due not only to evaporation into the air gap but also to superimposed effects of surface condensation, are very protracted, and on account of unavoidable small differences in initial temperatures and gap saturations, they are never reproducible either with respect to magnitude or variability with time. Such systems are, therefore, quite unavailable for protracted calorimetry and yield uncertain data even in quickly completed adiabatic measurements unless corrections be made for their variable leakage by careful interpolation from the results of preperiod and postperiod observations. The rates of leakage are, however, nearly enough linear during the first half hour to make the error of such interpolation negligible.

The open calorimeter with invisibly wetted outer surface, enclosed in a vapor saturated gap which holds no free water, exhibits equal or greater variability in the magnitude and character of the initial disturbances, in this case due to unavoidable differences in prior temperatures of calorimeter and bath. If the bath be previously lower, the calorimeter temperature, under conditions which normally establish an isothermal condition, slowly rises; if the bath be previously higher, it falls. Both of these changes are extremely protracted and may be shown to be caused by changes in the densities of the films of water adsorbed by the calorimeter surface. Under bath heads, both positive and negative, the leakage of systems of this type is greater than the corresponding leakage of the closed system through dry gaps of the same width. The excess leakage is probably caused partly by an increased convection rate, but in greater measure by distillation, that which occurs when the bath is at higher temperature being due to a transfer of moisture to the calorimeter from adsorbed films on the jacket walls. These open systems therefore are, like the

${ }^{40}$ Publications of the Etrest Kempton Adams Fund for Physical Research. 
preceding, unavailable for protracted measurements, though they may be similarly used in measurements of short duration. Although they involve greater and more variable leakages, they possess the advantage that under bath heads less than $0.2^{\circ}$ the leakage is the same in both directions, so that a correction for imperfect adiabaticity may be correctly determined on the assumption that leakage is proportional to thermal head. ${ }^{4} 7$

When the air spaces surrounding an open calorimeter hold free water, the leakages under both positive and negative bath heads are still further increased, primarily by the effects of free distillation. The phenomena in this case are so markedly affected by the quantity of water in the gap, by its location and distribution and by its influence on the magnitudes and rates of the surface adsorptions, that they exhibit at first glance a hopelessly capricious variability. A rough and partial analysis of these leakages was possible, but beyond furnishing additional data on the adsorption phenomena its only practical result was to prove that the presence of even a small quantity of water in the air gap might well invalidate any calorimetric determination.

As a whole the experiments with the open calorimeter led to the final judgment that although such systems may with proper precaution be used in quickly completed adiabatic measurements, they are unavailable for any other, and excepting for work confined within very narrow temperature ranges, would best be discarded altogether.

\section{Summary}

A. From the results of an extensive series of measurements undertaken to establish a standard of accuracy for the calorimetry of slow processes, it has been determined that in order to attain the accuracy defined by the gain or loss of half a gram-calorie in 10 hours, the following precautions are necessary and sufficient.

1. A closed calorimetric system must be used which allows no measurable evaporation into the insulating air gaps, and these gaps must be dry (unsaturated with water vapor).

2. The outer environment must be thermostatically controlled whenever there are conducting parts which pass directly from the calorimeter into the surrounding air, with a precision characteristic of the system employed. This is about $\pm 0.2^{\circ}$ when encased mercury thermometers are used. It is advisable to keep the environmental temperature always close to the calorimetric within the characteristic range of negligible di-

1" In the preceding research, which was carried out with an open system of this type, this proportionality was observed, and was made the basis of the correction for imperfect a.diabaticity. The present measurements confirmed these former observations, and by showing the probable causes of the proportionality, explained their seeming inconsistency with those of other observers, which was noted at the time. (Ref. 1, pp. 1923, 1924.) 
rect leakage; and it is necessary to correct for fluctuation beyond this range. A constant temperature closet is requisite for this control. A convenient construction of this sort is described.

3. With the usual system of about 1000 gram-calorie units heat capacity the air gaps should be not less than $40 \mathrm{~mm}$. wide: but there is no practical advantage in using gaps wider than this. The heat capacity of these wide gaps is relatively too small to cause indeterminate errors of measurement. With the $40 \mathrm{~mm}$. gap, when the bath temperature fluctuates within $\pm 0.01^{\circ}$, and the environment within $\pm 0.2^{\circ}$ of the calorimetric, the system is practically adiabatic within the error above defined, the total leakage, of which the convection factor is negligible, being little more than three times that of a perfect vacuum gap. The leakage rate in such systems is the same in both directions, and for heads below $0.1^{\circ}$ is proportional to the head. Correction for imperfect adiabaticity due to bath fluctuation may be made on this basis, since transmission lag has no measurable effect on the leakage. A new type of calorimeter embodying these advantages is described. With this system, the initial conditions are perfectly adiabatic; and the total calorimetric lag for the temperature changes characteristic of long-continued operations is negligible.

4. It is permissible and advantageous to stir the closed system reciprocally. In the installation described, this may be done without significant loss of heat. The heat of stirring is developed identically in different assemblages and at the same rate for any one speed, whether stirring is continuous or intermittent. It may vary at low speeds with the fourth and higher powers of the speed. The continuous stirring necessary to ensure mixture in chemically reacting systems is productive of negligible error; and similar stirring at low speeds throughout determination is shown to be practicable, though in very protracted operations intermittent stirring is the better practice. Strictly uniform speeds are necessary for long continuous stirring, but not for intermittent stirring, nor for continuous stirring during mixture if the approximate rates of heat production under different speeds be known for the system used.

5. The open calorimeter is unavailable in protracted calorimetry; since, even with an initially dry gap, evaporation leakage is inconstant as the result of uncontrollable variability in prior conditions. This is due in great part to the condensation of water vapor in the air gap on the walls of the calorimeter, which occurs on clean as well as on contaminated surfaces, and is probably due to the simple adsorption of moisture, an effect which can be neither eliminated nor controlled even under approximately isothermal conditions. With change of temperature its effects are greater and still more irregular, and in water-saturated gaps are complicated in the extreme.

B. The closed system is best for all types of calorimetry; but in 
the adiabatic measurement of swift reactions the open calorimeter may be safely used, either with dry surfaces in an initially dry gap, or with invisibly wetted surfaces in a saturated gap which holds no water, provided always that correction for aggregate thermal disturbances due to evaporation leakage be made by interpolation from the results of observations taken before and after the reaction period. The initially dry gap, with which leakage is less, and more nearly uniform, is preferable in such measurement; but correction for imperfect adiabaticity is safer with the saturated gap.

Under all heads less than $1^{\circ}$, the total leakage from a closed calorimetric system in a dry gap shows slight divergence irom Newton's law, since the convection increases very slowly with increase in gap width, and varies as powers of the thermal head less than 2 excepting when the gap is unusually wide.

Similar leakage from the open system is irregular, but yields itself to a rough analysis which gives some indication of the character of the thermal disturbances and the magnitude of the uncertainties characteristic of the merodiabatic measurements which have yielded the bulk of our present thermochemical data.

An experimental method for the determination of conduction leakage in any closed calorimetric system is described, and a simple empirical rule for estimating its approximate magnitude in closed cylindrical systems of convenient dimensions is given.

As a whole the work illustrates several applications of an experimental procedure which permits the long-continued maintenance either of an adiabatic condition or of a condition of constant leakage in a system of changing temperature. The latter condition is available for isothermal calorimetry, and for the simplification of method in other types of physical measurement. In illustration of one such application, the thermal conductivity of air, incidentally determined (with no specific precautions) at atmospheric pressure by the analysis of thermal leakage including convection yielded $k_{20}{ }^{\circ}=0.000057$, in close agreement with the accepted value.

Precise data are submitted which quantitatively define the above statements.

In conclusion, the writer desires to express his obligation to the Department of Physics of Columbia University for laboratory accommodations and shop facilities essential to the prosecution of this investigation, and to the University for the Ernest Kempton Adams Research Fellowship Fund, with which the expenses incident to it were defrayed.

NEW YoRK, N. Y. 